\title{
3D Monte Carlo simulation of backscattered electron signal variation across pore-solid boundaries in cement-based materials
}

\author{
M.H.N. Yio, H.S. Wong" and N.R. Buenfeld \\ Concrete Durability Group, Department of Civil and Environmental Engineering, Imperial College London, SW7 2AZ, \\ $U K$
}

\begin{abstract}
Three-dimensional (3D) Monte Carlo simulation was used to study the variation of backscattered electron (BSE) signal across pore-solid boundaries in cement-based materials in order to enhance quantitative analysis of pore structure. The effects of pore size, depth and boundary inclination angle were investigated. It is found that pores down to $1 \mathrm{~nm}$ can generate sufficient contrast to be detected. Visibility improves with larger pore size, smaller beam probe size and lower acceleration voltage. However, pixels in shallow pores or near pore boundaries display higher grey values (brightness) than expected due to sampling sub-surface or neighbouring solid material. Thus, cement-based materials may appear less porous or the pores appear smaller than they actually are in BSE images. Simulated BSE images were used to test the accuracy of the Overflow pore segmentation method. Results show the method is generally valid and gives low errors for pores that are $1 \mu \mathrm{m}$ and greater.
\end{abstract}

Keywords: Backscattered electron imaging; Pore structure; Cement-based materials; 3D Monte Carlo simulation; Image analysis; Pore segmentation.

\section{Introduction}

The microstructure of cement-based materials plays a critical role in controlling the performance of concrete structures. In particular, the pores (and cracks) inherent in the microstructure influences the durability of concrete structures as they provide channels for ingress of deleterious species (such as chloride ions, carbon dioxide, sulphate ions etc.) causing a range of degradation mechanisms. The pore structure, which ranges over six orders of magnitude from nanometre to millimetre, also controls strength, elasticity and other important engineering properties such as creep and shrinkage. As such, there is a huge interest in characterising the pore structure of concrete.

Backscattered electron (BSE) microscopy has long been established as a versatile technique for quantitative characterisation of concrete microstructure. This is because the technique is capable of providing actual images of the microstructure at very high resolution and allows different phases to be distinguished based on their brightness [1]. Phase brightness is a function of the collected BSE coefficient, which increases monotonically with mean atomic number of the phase. Hence, in a BSE image of epoxy-impregnated polished cement paste, the unreacted cement particles appear the brightest, followed by hydration products such as calcium hydroxide $(\mathrm{CH})$ and calcium silicate hydrate (C-S-H), while the epoxy-filled pores and cracks appear the darkest. Some applications of quantitative BSE imaging in cement and concrete research include measuring reaction degrees, estimating mix composition and assessing deterioration. Quantitative BSE microscopy has also been used to characterise many other types of porous materials including bone [2], rocks [3] and alloys [4].

Two critical aspects of quantitative microscopy are the accuracy of feature segmentation and resolution, i.e. the smallest feature that can be reliably measured. Segmentation is usually carried out by selecting the appropriate upper and lower grey level threshold values from the brightness histogram that correspond to the phase of interest. However, the process can be ambiguous and prone to error [5]. This is partly because pixels near boundaries tend to exhibit gradual transition in grey values due to mixing of signals from neighbouring phases. As a result, different phases may share similar grey values making it very difficult to define the thresholds that can satisfy all boundary conditions. In quantitative microscopy, it also important to know the size of the smallest feature that can be reliably imaged and measured. This not only defines the capability of a particular instrument/technique, but also the accuracy and potential errors of the measurement. Furthermore, understanding factors that influence resolution helps optimisation of the imaging technique for a particular application.

Monte Carlo simulation of electron-solid interactions offers a unique means to study signal transition across phase boundaries $[6,7]$ where experiments would not be possible. Such simulations could help improve image segmentation and establish the theoretical resolution for a particular phase of interest. The Monte Carlo technique uses a stochastic process to simulate the elastic and inelastic scattering of electrons in any solids and across any boundary types. Each electron trajectory is monitored in a stepwise manner from its entry point until the electron is either absorbed by the

\footnotetext{
* Corresponding author. Tel: +44 (0)20 7594 5956; E-mail: hong.wong@imperial.ac.uk
} 
sample or backscattered. This technique has been developed over the last five decades to provide a theoretical foundation underpinning electron microscopy and X-ray microanalysis, and to assist quantitative interpretation of SEM images. Details of the physics behind the technique can be found in [8] and [9].

In cement and concrete research, Wong and Buenfeld [10] have carried out Monte Carlo simulations to study the shape and size of the interaction volume, spatial and energy distribution of backscattered electrons and characteristic X-rays in cement-based materials. However, the study was limited to two-dimensional simulations of single phases and to tungsten thermionic emitters that have now been surpassed by field emitters. In this paper, we present three-dimensional Monte Carlo simulations to investigate how BSE signal varies across pore-solid boundaries in cement-based materials. A range of pore size, depth and orientation were simulated. Other variables included emitter type, beam accelerating voltage and probe diameter. The aim of the work was to better understand signal transition across pore-solid boundaries in order to enhance quantitative analysis of pore structure. Results were used to establish the resolution of BSE microscopy for pore analysis and to test accuracy of the Overflow pore segmentation method [11].

\section{Simulation}

\subsection{D Monte Carlo simulation}

3D CASINO (Version 3.2) was used to perform the simulations throughout this study. The Monte Carlo simulation software is an update of the 2D version developed by [12] and [13]. A comprehensive description of the software is given in [14]. In the current version, electron trajectories can be traced in three-dimensions in complicated models built from basic shapes and planes. This allows pore structure of different configurations to be investigated. Another important feature is the ability to perform areal scanning to generate realistic BSE images. This is particularly useful for testing and verifying quantitative image analysis. The software allows users to choose various simulation settings including the physical model, number of electrons, angle of incident beam, accelerating voltage, probe diameter etc. Furthermore, the accuracy of the software has been validated by [14] based on a comparison between simulated and measured backscattered coefficients of a silicon sample at beam energies below $5 \mathrm{keV}$.

In this study, the Mott model and the modified Bethe equation were adopted for modelling elastic scattering, and deceleration and energy loss of electrons respectively. In order to ensure that the obtained results were statistically significant, a large number of electrons was simulated per analysis. Unless otherwise stated, $4 \times 10^{5}$ electrons were simulated per spot for point and line scans. This yields a relative error of $0.16 \%\left(\approx 1 / n^{0.5}\right.$, where $n$ is the number of electrons). For areal scans, the number of electrons was halved to $2 \times 10^{5}$ per spot to reduce computation time, but still keeping a small relative error of $0.22 \%$. The computational time for a typical simulation consisting of 124 points using $4 \times 10^{5}$ electrons at $10 \mathrm{keV}$ was approximately $5.5 \mathrm{~h}$ on a workstation (Intel ${ }^{\circledR}$ Xeon ${ }^{\circledR}$ CPU E5-1650, 3.2 GHz processor). The angle of the incident beam was set perpendicular to the sample surface since this is the most common configuration for quantitative BSE imaging. The trajectory of each electron was traced until its energy fell below $50 \mathrm{eV}$ or until it left the sample surface. The probe diameters used and their corresponding accelerating voltages are presented in the following Section.

\subsection{Probe diameter}

Four different types of emitter were simulated in this investigation to cover the range of emitters available in practice. These were tungsten and lanthanum hexaboride thermionic, Schottky and cold field emitters. The probe diameter for each emitter was derived based on the method proposed by [15]. The method uses practical brightness, which determines the actual amount of current in the probe, to calculate the source image size $\left(d_{I}\right)$. The total probe diameter $\left(d_{p}\right)$ was obtained by adding $d_{\mathrm{I}}$ together with other contributions including diffraction $\left(d_{A}\right)$, chromatic $\left(d_{C}\right)$ and spherical $\left(d_{S}\right)$ aberrations using the root-power-sum (RPS) method as shown in Eq. (1). In order to eliminate assumptions concerning the electron probe profile, the full width median (FW50) values were adopted for all contributions. Further explanations of this are given by [15].

$$
d_{p}=\sqrt{\left[\left(d_{A}^{4}+d_{S}^{4}\right)^{1.3 / 4}+d_{I}^{1.3}\right]^{2 / 1.3}+d_{C}^{2}}
$$

Table 1 shows the calculated probe diameters for all emitters at increasing beam energies of 5, 10, 15, 20, 25 and 30 $\mathrm{keV}$. The calculated probe diameters ranged between 1 and $150 \mathrm{~nm}$, and decreased with increasing beam energy as expected. Field emitters produced the brightest source and smallest probe diameters. Detailed calculations and assumptions involved are presented in Appendix I.

\subsection{D models of pore-solid boundaries in cement-based materials}

A total of 119 simulations representing a range of pore sizes and geometries were carried out. The pores were assumed to be filled with a low viscosity araldite resin $\left(\mathrm{C}_{10} \mathrm{H}_{18} \mathrm{O}_{4}\right)$ of $1.14 \mathrm{~g} / \mathrm{cm}^{3}$ specific gravity. This is because samples are 
usually impregnated with resin to preserve the delicate microstructure and produce atomic contrast for BSE imaging. Calcium silicate hydrate (C-S-H) was taken to represent the solid since this is the main binding phase and hydration product forming in the originally water-filled spaces during cement hydration. However, simulating the C-S-H phase is challenging because it has variable composition and disordered structure [16-19]. For simplicity, the general formula $x \mathrm{CaO} . \mathrm{SiO}_{2} \cdot y \mathrm{H}_{2} \mathrm{O}$ was used. The $\mathrm{Ca} / \mathrm{Si}$ ratio of C-S-H in hardened cement pastes generally range between 1.2 and 2.3 , with the mean value close to 1.75 [17]. $\mathrm{The}_{2} \mathrm{O} / \mathrm{SiO}_{2}$ ratio and $\mathrm{C}-\mathrm{S}-\mathrm{H}$ density depend on moisture state. However, high-resolution BSE imaging is usually performed on dried samples in vacuum. For C-S-H with a monolayer of water at $11 \%$ relative humidity, an approximated chemical composition of $1.7 \mathrm{CaO} \cdot \mathrm{SiO}_{2} \cdot 2.1 \mathrm{H}_{2} \mathrm{O}$ and specific gravity of 2.47 $\mathrm{g} / \mathrm{cm}^{3}$ have been suggested [19]. These values were used throughout the study and it is assumed that the variation in electron scattering characteristics and the resulting BSE signals within various forms of C-S-H is small and insignificant compared to the variation across the pore-solid boundary.

3D models of three basic pore-solid configurations were generated for the simulations (Figure 1). The first model (A) consists of a vertical pore (epoxy-filled) intercalated between two C-S-H blocks of indefinite size. The pore size $(x)$ was varied from $1 \mathrm{~nm}$ to $10 \mu \mathrm{m}$ to cover the range of "gel" and "capillary" pores in cement-based materials. This model was used to study the effect of pore size on BSE signal variation and to determine the smallest resolvable pore in BSE imaging. The second model (B) consists of two configurations: a pore overlying a C-S-H layer and another in the reverse order. The thickness of the top layer $(y)$ was increased gradually from 0 to $8 \mu \mathrm{m}$ to investigate the effect of pore depth and sampling of subsurface material on the recorded BSE signal. The third model (C) comprises of adjoining pore and C-S-H layers inclined at angle $\theta$ ranging from $10^{\circ}$ to $170^{\circ}$. This was used to study how inclination angle of the poresolid boundary affects the transition of BSE signal.

The size of all three models was set to be at least ten times larger than the interaction volume of the electrons to ensure that all interactions occurred within the model and all backscattered electrons were captured. It was assumed that the sample surface was perfectly flat, each phase was stoichiometric and homogeneous, and the interface between the pore and C-S-H phases was abrupt.

\section{Results}

\subsection{Effect of pore size on BSE signal variation}

Figure 2 shows the variation in the simulated BSE coefficient obtained by line scans across the Model A shown in Figure 1. Simulations were carried out for pore sizes of $1 \mathrm{~nm}, 10 \mathrm{~nm}, 100 \mathrm{~nm}, 1 \mu \mathrm{m}$ and $10 \mu \mathrm{m}$, at 10 and $20 \mathrm{keV}$ beam energies for five emitter types based on the data shown in Table 1. Note that the extent of all scans (linear dimension) was at least $\pm 5 \times$ the pore size from the centre of the pore to reduce sample edge effects. The spacing between each scan point (i.e. pixel spacing) was set to be one tenth of the pore size to ensure that sufficient information was captured.

Overall, the amount of BSE signal variation across the pore-solid boundary increases with increase in pore size. The BSE coefficient of the pore decreases with increase in pore size and only achieves the correct value for pure epoxy $(\sim 0.045-0.047)$ when the pore size is $100 \mathrm{~nm}$ or greater. This is because of the large sampling volume of BSE relative to the pore size causing the recorded signals from small pores to be affected by adjacent C-S-H. This effect increases with acceleration voltage. For example, the maximum penetration depth and escape radius of BSE in pure epoxy (average of five simulations) are 1.0 and $1.9 \mu \mathrm{m}$ respectively at $10 \mathrm{keV}$ and 3.4 and $6.6 \mu \mathrm{m}$ respectively at $20 \mathrm{keV}$ (Figure $3 \mathrm{a}$ ). In C$\mathrm{S}-\mathrm{H}$, these values are 0.6 and $1.0 \mu \mathrm{m}$ respectively at $10 \mathrm{keV}$ and 1.8 and $3.6 \mu \mathrm{m}$ respectively at $20 \mathrm{keV}$ (Figure $3 \mathrm{~b}$ ). The interaction volume of electrons in epoxy is almost $3.5 \times$ larger than in C-S-H as the mean atomic number of epoxy (6.184) is much lower than that of C-S-H (12.086) [10].

For a $1 \mathrm{~nm}$ pore, the field emitters are able to detect a slight signal variation across the pore whereas the thermionic emitters detect no signal variation. This is simply because the thermionic emitters have probe diameters much larger than the pore thus a large proportion of the incident electrons enter and backscatter directly from the C-S-H. For $10 \mathrm{~nm}$ and $100 \mathrm{~nm}$ pores, all emitters show improvements in the detected signals, but significant differences between the performance of field and thermionic emitters are still evident. At $1 \mu \mathrm{m}$ and above, all emitters show similar levels of BSE signal variation. It is also noted that transition of the BSE coefficient is not abrupt, but occurs over a finite distance (up to $500 \mathrm{~nm}$ in some cases) as the beam scans across the pore-solid boundary. For large pores, there is a slight increase in the BSE signal on the C-S-H side as the beam approaches the pore-solid boundary. This is an edge effect due to strong scattering of electrons from the high atomic C-S-H into the low atomic epoxy-filled pore that consequently has greater probability of escaping the sample. An opposite effect occurs on the pore side of the boundary causing a slight dip in the BSE signal.

\subsection{Contrast and visibility of pores}

Since the BSE signal variation decreases with decrease in pore size, there will be a limit when the signal variation is too small to be discernable. This would represent the spatial resolution or detection limit for imaging pores. One useful way to quantify the visibility of the pore is to calculate the contrast between the pore and adjacent C-S-H using Eq. (2). 


$$
C=\frac{\eta_{1}-\eta_{2}}{\eta_{1}} \times 100, \eta_{1}>\eta_{2}
$$

Where $\eta_{1}$ and $\eta_{2}$ are the BSE coefficients of the C-S-H and at the centre of the pore respectively.

Figure 4 (a) and (b) show the calculated BSE contrast as a function of the pore size and probe diameter respectively. The signal contrast increases with increase in pore size, but reduces with increasing probe diameter (when the pore size is smaller than the probe diameter). The theoretical contrasts between pure epoxy $\left(\eta_{2} \sim 0.045-0.047\right)$ and pure C-S-H $\left(\eta_{1} \sim 0.142-0.143\right)$ at 10 and $20 \mathrm{keV}$ are approximately $67 \%$ and $68 \%$ respectively. Such levels of contrast are only observed in the simulations for pores $100 \mathrm{~nm}$ or greater at $10 \mathrm{keV}(1000 \mathrm{~nm}$ at $20 \mathrm{keV})$. As expected, the contrast at 20 $\mathrm{keV}$ is lower than at $10 \mathrm{keV}$. The contrast peak at $1000 \mathrm{~nm}$ is likely to be due to the drop in BSE signal at the centre of the pore caused by edge effects at the boundaries. Assuming that the minimum contrast required for visibility is $5 \%$ [9], the simulations suggest that a pore as small as $10 \mathrm{~nm}$ (1 nm in some cases) can generate sufficient contrast to be detected. This is true despite the fact that the pore size is substantially smaller than the BSE sampling volume (Figure $3)$.

Figure 5 shows the simulated BSE images for Model A to study the effect of pore size, emitter type and beam energy on visibility. The simulated BSE coefficients were converted into grey values that stretch across the 8 -bit grey scale $(0$ to 255) using the mat2gray and im2uints functions in MATLAB®. A grey value of " 0 " represents the BSE coefficient of pure epoxy whereas a grey value of " 255 " represents the BSE coefficient of tetracalcium aluminoferrite $\left(\mathrm{C}_{4} \mathrm{AF}\right)$, which is the brightest phase present in unreacted cement in hardened cement paste. This was to give the simulated pore and C$\mathrm{S}-\mathrm{H}$ phases grey values that resemble those from real BSE images of cement paste. The pixel spacing is one tenth of the pore size and the image size is $100 \times 25$ pixels; the total pore fractions in all images are therefore exactly $10 \%$. There are a few interesting observations to be made from these figures. First, in agreement with the results in Figure 2 and 4, a pore size as small as $1 \mathrm{~nm}$ is indeed detectable with field emitters; however, its visibility is poor. Second, the boundary between pore and C-S-H appears fuzzy for pores smaller than the probe diameter. Third, the contrast between pore and $\mathrm{C}-\mathrm{S}-\mathrm{H}$ is noticeably higher at $10 \mathrm{keV}$ than at $20 \mathrm{keV}$. Overall, as already discussed above, these conditions improve with increasing pore size and decreasing probe diameter and accelerating voltage. From the following section onwards, all simulations were performed using the Schottky field emitter since it is a commonly used emitter in modern SEMs and offers good resolution.

\subsection{Effect of sampling subsurface material}

Figure 6 (a) shows the change in BSE coefficient as the thickness (depth) of the epoxy-filled pore layer overlying the CS-H layer (Model B, Figure 1) increases from 0 to $7 \mu \mathrm{m}$. Figure 6 (b) shows the results for the reverse configuration (C$\mathrm{S}-\mathrm{H}$ layer overlying pore). The accelerating voltage was increased from 5 to $30 \mathrm{keV}$ at $5 \mathrm{keV}$ intervals to investigate the effect of the interaction volume on sampling subsurface material. The simulated BSE coefficient has a marginal dependency on accelerating voltage. This is a well-known phenomenon, but the exact relationship between the BSE signal and accelerating voltage is complex. However, the variation in the BSE coefficient within the accelerating voltage range of 5 to $50 \mathrm{keV}$ is generally less than $10 \%$ [9].

Results for both configurations demonstrate that when the thickness of the top layer decreased beyond a critical value, the BSE coefficient changes gradually to that of the bottom layer material. This is because the electron beam penetrates the top layer and samples the bottom layer. This critical thickness is approximately the maximum penetration depth of the backscattered electrons of the top layer. The resulting BSE pixel brightness is therefore not that of the top layer. As expected, the critical thickness increases with accelerating voltage. For a pore/C-S-H configuration, the critical thickness range from $0.3 \mu \mathrm{m}$ at $5 \mathrm{keV}$ to $6.6 \mu \mathrm{m}$ at $30 \mathrm{keV}$. For a C-S-H/pore configuration, the critical thickness ranges from $0.2 \mu \mathrm{m}$ at $5 \mathrm{keV}$ to $3.2 \mu \mathrm{m}$ at $30 \mathrm{keV}$. Unsurprisingly, the critical thickness is larger when the pore is overlying CS-H.

Figure 7 shows simulated BSE images of an epoxy-filled pore that is overlying C-S-H at 10 and $20 \mathrm{keV}$. The images are $10 \times 10$ pixels at $0.1 \mu \mathrm{m}$ spacing. The simulated BSE coefficients were converted to pixel grey values and stretched across the 8-bit grey scale (see Section 3.2 for explanation). Results show that the grey value of a pore can spread over a range depending on its depth due to sampling of underlying C-S-H. Pores shallower than the critical thickness would share similar grey value as the solid C-S-H. The opposite effect could occur if a thin C-S-H layer overlies a large pore, but this is expected to be less severe because the penetration depth of BSE in C-S-H is less than half of that in epoxy. The implication of this is that cement-based materials with very fine pore sizes would appear to be denser (i.e. having lower porosity) than they are. Pores may appear smaller than they actually are due to the transition in grey value near boundaries. Undoubtedly, this effect increases the ambiguity concerning the true position of the pore-solid boundary, creates errors during pore segmentation and limits the smallest pore size that can be reliably imaged. 
In reality, the pore-C-S-H boundaries are not perpendicular to the sample surface, but occur at varying angles because of their complex and irregular morphology. Figure 8 shows the effect of this on the BSE coefficient measured by line scans across Model C (Figure 1) where the pore-C-S-H boundary angle varied from $10^{\circ}$ to $170^{\circ}$. The scans were up to \pm $20 \mu \mathrm{m}$ from the pore-C-S-H boundary. The position of the first and last point of each scan was such that their distances to the boundary were greater than the BSE escape surface radius of epoxy and C-S-H respectively. The spacing between each point was $20 \mathrm{~nm}$ at $10 \mathrm{keV}$ and $100 \mathrm{~nm}$ at $20 \mathrm{keV}$.

Figures 9 (a), (b) and (c) show the change in the shape and size of the interaction volume of electrons across the pore-CS-H boundary at $10^{\circ}, 90^{\circ}$ and $170^{\circ}$ respectively at $10 \mathrm{keV}$. As the incident beam approaches the boundary, electrons begin to sample the neighbouring higher atomic number C-S-H phase and the interaction volume diminishes in size. The changes in size and shape of the interaction volume, and in the resulting BSE coefficients are more abrupt when the boundary is perpendicular to the surface, but occur more gradually with shallow sloping boundaries $\left(10^{\circ}\right.$ and $\left.170^{\circ}\right)$ due to sampling of subsurface material. This effect is more severe on the pore phase where the BSE signal transition can occur over a distance of $5 \mu \mathrm{m}$ at $10 \mathrm{keV}$ and $16 \mu \mathrm{m}$ at $20 \mathrm{keV}$ for a $10^{\circ}$ slope (Figure 8).

Figure 10 shows the simulated BSE images of pore-C-S-H boundaries at various inclination angles at $10 \mathrm{keV}$. The image size is $1800 \times 120$ pixels at $0.1 \mu \mathrm{m}$ spacing. The pore fraction in all images is $50 \%$. It can be seen that the pixels near sloping boundaries exhibit a gradient in grey value and that the effect is stronger on the pore phase. This clearly presents a significant challenge in determining the true position of the pore-C-S-H boundary. In manual thresholding, one is likely to assume that the darkest pixels represent the pore phase. This would under-estimate the size of pores at angles $<90^{\circ}$ and over-estimate at angles $>90^{\circ}$.

\subsection{Pore segmentation via image analysis}

As mentioned in the Introduction, one of the main motivations for this study was to test the accuracy of the Overflow method [11] for segmenting pores in cement-based materials. The simulated BSE images in the previous sections serve as a useful tool for this purpose. In the Overflow segmentation method, the upper threshold grey value for pores is determined from the inflection point in the cumulative brightness histogram of the BSE image. This represents a critical point at which the segmented pore areas start to 'overflow' into the surrounding solid paste matrix. The method provides a consistent means for phase segmentation and it has since been used in a number of applications including clinker studies [20], characterising microstructure development and interfaces [21-25], determining original water/cement ratio and mix composition [26], and mass transport modelling [27, 28].

Figure 11 shows the cumulative brightness histograms of the simulated BSE images from Sections $3.1 \& 3.2$ (from Figure 5). It can be seen that the simulated curves exhibit a sigmoidal behaviour similar to those from real BSE images of cement-based materials. The sigmoidal trend increases with increase in contrast and sharpness of the pore-solid boundary. Note that the results from a $1 \mathrm{~nm}$ pore obtained by the tungsten and lanthanum hexaboride emitters, and from a $10 \mathrm{~nm}$ pore obtained by the tungsten emitter were excluded from the analysis as these images do not contain sufficient signal to resolve the pore phase (Figure 5).

The inflection points determined by the Overflow method are marked with crosses while the correct grey values that correspond to the exact pore area fractions are marked with circles. The results show that when pores are smaller than 1 $\mu \mathrm{m}$, the inflection points overestimate the correct grey values with significant errors. Note that these errors are not due to digitisation effects since the pixel size in the simulations were set at one-tenth of the pore size. For pores of $1 \mu \mathrm{m}$ and greater, the inflection points generally agree very well with the correct grey values (Figure $11 \mathrm{~d}$ and e) in particular for field emitters at $10 \mathrm{keV}$. Figure 12 compares the overflow segmented pore size with the actual pore size. It can be seen that the agreement between measured and actual values improves with increase in pore size. For pores of $1 \mu \mathrm{m}$ and greater, errors of $\sim 1 \%$ were observed for field emitters at $10 \mathrm{keV}$. The results suggest that the minimum pore size that can be accurately segmented by the Overflow method is approximately half the BSE escape radius in epoxy, which is 1 $\mu \mathrm{m}$ and $3 \mu \mathrm{m}$ at $10 \mathrm{keV}$ and $20 \mathrm{keV}$ respectively for field emitters.

Figure 13 shows the cumulative brightness histograms and pore segmentation errors for the simulated images from Section 3.4 (from Figure 10) with inclined pore-solid boundaries. The segmentation is very accurate for pores with < $90^{\circ}$ inclination angles. However, segmentation errors increase for inclination angles larger than $90^{\circ}$, up to a value of $30.7 \%$ at $170^{\circ}$. The over-estimation is due to presence of dark pixels on the C-S-H side of the boundary from sampling subsurface pore (Figures $9 \mathrm{c} \& 10$ ). Assuming that pores are randomly orientated and that the probability of the pore occurring at any angle is equal, the error contribution from each inclination angle can be averaged to determine the overall percentage error of the segmentation. The resulting value is $5.2 \%$ and this indicates that the total pore fraction would be marginally overestimated with the Overflow method. 


\section{Discussion}

Electron scattering near phase boundaries is a complex process. This is particularly true if the phases have large differences in atomic number and density since the interaction volume from which useful signals emerge will vary on both sides of the interface. Near boundaries, scattered electrons can move from one phase to another and this influences the collected signals and images. For BSE imaging of cement-based materials, samples are usually impregnated with a low atomic number material (epoxy) to generate high contrast between pores (or cracks) and solids. This enhances visibility and facilitates quantitative characterisation via image analysis.

However, the large interaction volume in epoxy-filled pores increases the likelihood of sampling sub-surface or neighbouring solid phases. This can be problematic because the pores (gel and capillary) in cement-based materials range from sub-micron to several microns in size and the pore boundaries with solid hydration products have complex morphologies. This study shows that the signal measured across pore boundaries may vary over a distance of several microns. Pixels in shallow pores or near boundaries will display grey values (brightness) higher than expected because of additional scattering events occurring into the solid hydration products. This adds uncertainty concerning the exact location of the pore-solid boundary for segmentation and image analysis. It also means that the sample may appear less porous or the pores appear smaller than they actually are in BSE images.

As shown in this study, the spatial resolution of BSE imaging for pore characterisation is influenced by the probe size, signal sampling volume and interactions that occur near phase boundaries. These are dependent on the emitter type, beam energy and composition of the phases present. The fact that pores smaller than the BSE sampling volume or the electron probe size are visible (Figure 5) shows that these dimensions do not represent the spatial resolution limit. This is because phase visibility depends on the difference between the BSE signal detected from that particular phase and its neighbouring region, i.e. contrast. Features smaller than the BSE sampling volume or probe size can still be detected as long as the obtained contrast satisfies the visibility criteria.

Since the collected images are digitised, the pixel size may also influence final resolution. Pixel size decreases with increase in magnification. For images captured at low magnification, spatial resolution is likely to be limited by the pixel size. However, at high magnification, the influence of pixel size becomes less important especially when it is much smaller than the signal sampling volume or the electron probe diameter. This is because of overlapping signals from adjacent pixels. Therefore, pixel size is not always equivalent to the image resolution. The actual resolution can be no better than the pixel size, but is generally less due to the various factors discussed above.

It is important to note that the results presented in this paper represent ideal imaging conditions. In reality, the spatial resolution could be worse. This is because the effect of noise was not considered in the simulations. Signal noise can come from uneven surface topology and geometry of the sample, poor efficiency of the detector, shot noise from the electron beam, insufficient number of electrons collected due to high scan speed, inadequate imaging environment and signal digitisation process etc. These factors will further complicate the BSE signal generation across the pore-C-S-H boundary. In real cement-based materials, pores can also occur adjacent to other solid phases such as calcium hydroxide $(\mathrm{CH})$ and unreacted cement phases. In particular, 'Hadley' grains, which consist of darker rims (mixture of porosity and low-density C-S-H) around unreacted cement, can often be present in the microstructure of cement paste [29-31]. Both $\mathrm{CH}$ and unreacted cement have higher atomic number than C-S-H and would increase the BSE coefficient at the pore boundary. However, the general behaviour of the signal transition across the pore boundary should be similar to that observed in this study.

Although pores smaller than the BSE sampling volume or the electron probe size may be visible (Figure 5), significant measurement errors could occur during quantitative image analysis. The magnitude of this error would depend on the size of the pore with respect to the image resolution and the accuracy of the segmentation method employed.

Segmentation is one of the most important, but error prone, processes in quantitative microscopy. The Overflow segmentation method was shown to be accurate for segmenting pores that are larger than half the escape radius of backscattered electrons. The Overflow method is also able to determine the boundary of shallow pores overlying C-S-H and inclined pores with reasonably low errors. Thus, the method seems robust and handles grey scale variation at poresolid boundaries well. Nevertheless, it has to be stressed that no segmentation method is perfect and that some degree of error will always occur during image analysis, particularly for heterogeneous, multi-phase and multi-scale materials. The magnitude and significance of this error should always be considered in quantitative image analysis.

\section{Conclusion}

A 3D Monte Carlo technique was used to study the variation of backscattered electron (BSE) signal across pore-solid (C-S-H) boundaries in cement-based materials. The simulated pores were epoxy-filled and covered a range of sizes (1 $\mathrm{nm}$ to $10 \mu \mathrm{m})$, depths $(0$ to $7 \mu \mathrm{m})$ and inclination angles $\left(10^{\circ}\right.$ to $\left.170^{\circ}\right)$. Other variables included emitter type (tungsten thermionic, $\mathrm{LaB}_{6}$, Schottky and tungsten cold field), accelerating voltage (5 to $30 \mathrm{keV}$ ) and probe diameter (1 to 150 $\mathrm{nm})$. The main findings are: 
a) A pore size of $1 \mathrm{~nm}$ can generate sufficient contrast at $10 \mathrm{keV}$ using Schottky or cold field emitters to allow detection, despite the pore itself being smaller than the beam interaction volume. However, the visibility of $1 \mathrm{~nm}$ pores is poor. The visibility improves with increasing pore size, but degrades with increasing probe diameter and accelerating voltage.

b) Pores pixels can appear brighter (i.e. having larger grey value) than expected in BSE images due to sampling of sub-surface or neighbouring solid material. This occurs in shallow pores where the depth to underlying solid C-S$\mathrm{H}$ is less than the maximum penetration depth of BSE in epoxy $(\sim 1.0 \mu \mathrm{m}$ at $10 \mathrm{keV}$ and $3.4 \mu \mathrm{m}$ at $20 \mathrm{keV})$. This also occurs when pore pixels are located within the BSE escape radius in epoxy $(\sim 1.9 \mu \mathrm{m}$ at $10 \mathrm{keV}$ and $6.6 \mu \mathrm{m}$ at $20 \mathrm{keV}$ ) from the boundary.

c) These effects may cause misinterpretation of BSE images of heterogeneous porous materials. Pores in cementbased materials may appear smaller than they actually are due to the transition in grey value near boundaries from sampling sub-surface or neighbouring solids. This increases the uncertainty concerning the true position of poresolid boundaries, creates potential errors during segmentation and further limits the smallest pore that can be reliably measured.

d) Monte-Carlo simulated BSE images were used to test the accuracy of the Overflow pore segmentation method. Results show that the cumulative brightness histograms of the simulated images exhibit a sigmoidal behaviour similar to real BSE images of cement-based materials. The inflection point in the cumulative brightness histogram provides a good estimate for the threshold grey value for pore segmentation. For pore sizes of $1 \mu \mathrm{m}$ and greater, the Overflow method gave errors of $\sim 1 \%$ with field emitters at $10 \mathrm{keV}$. For inclined pores with random orientation, the average segmentation error was $5.2 \%$.

\section{Acknowledgements}

M.H.N. Yio would like to acknowledge the Dixon scholarship awarded by the Department of Civil and Environmental Engineering, Imperial College London.

\section{References}

[1] K.L. Scrivener, Backscattered electron imaging of cementitious microstructures: understanding and quantification, Cement and Concrete Composites, 26 (2004) 935-945.

[2] A. Boyde, J.C. Elliott, S.J. Jones, Bone Morphometry 1992 Sixth International Congress ProceedingsStereology and histogram analysis of backscattered electron images: Age changes in bone, Bone, 14 (1993) 205-210.

[3] A. Dilks, S. Graham, Quantitative mineralogical characterization of sandstones by back-scattered electron image analysis, Journal of Sedimentary Research, 55 (1985).

[4] E.J. Payton, P.J. Phillips, M.J. Mills, Semi-automated characterization of the phase in Ni-based superalloys via highresolution backscatter imaging, Materials Science and Engineering: A, 527 (2010) 2684-2692.

[5] J.C. Russ, The image processing handbook, 6th ed., CRC Press, London, 2011.

[6] D.R. Cousens, D.C. Joy, A monte carlo study of the position of phase boundaries in backscattered electron images, Scanning, 19 (1997) 547-552.

[7] C. Probst, H. Demers, R. Gauvin, Spatial Resolution Optimization of Backscattered Electron Images Using Monte Carlo Simulation, Microscopy and Microanalysis, 18 (2012) 628-637.

[8] D.C. Joy, Monte Carlo modeling for electron microscopy and microanalysis, Oxford University Press, New York, 1995.

[9] J. Goldstein, D.E. Newbury, D.C. Joy, C.E. Lyman, P. Echlin, E. Lifshin, L. Sawyer, J.R. Michael, Scanning electron microscopy and X-ray microanalysis, Springer, 2003.

[10] H.S. Wong, N.R. Buenfeld, Monte Carlo simulation of electron-solid interactions in cement-based materials, Cement and Concrete Research, 36 (2006) 1076-1082.

[11] H.S. Wong, M.K. Head, N.R. Buenfeld, Pore segmentation of cement-based materials from backscattered electron images, Cement and Concrete Research, 36 (2006) 1083-1090.

[12] P. Hovington, D. Drouin, R. Gauvin, CASINO: A new monte carlo code in C language for electron beam interaction - part I: Description of the program, Scanning, 19 (1997) 1-14.

[13] D. Drouin, A.R. Couture, D. Joly, X. Tastet, V. Aimez, R. Gauvin, CASINO V2. 42 - A Fast and Easy-to-use Modeling Tool for Scanning Electron Microscopy and Microanalysis Users, Scanning, 29 (2007) 92-101. 
[14] H. Demers, N. Poirier-Demers, A.R. Couture, D. Joly, M. Guilmain, N. de Jonge, D. Drouin, Three-dimensional electron microscopy simulation with the CASINO Monte Carlo software, Scanning, 33 (2011) 135-146.

[15] M.S. Bronsgeest, J.E. Barth, L.W. Swanson, P. Kruit, Probe current, probe size, and the practical brightness for probe forming systems, Journal of Vacuum Science \& Technology B, 26 (2008) 949-955.

[16] J.F. Young, W. Hansen, Volume Relationships for C-S-H Formation Based on Hydration Stoichiometries, MRS Online Proceedings Library Archive, 85 (1986) 313 (310 pages).

[17] I.G. Richardson, The nature of C-S-H in hardened cements, Cement and Concrete Research, 29 (1999) $1131-1147$.

[18] I.G. Richardson, Electron microscopy of cements, in: J. Bensted, P. Barnes (Eds.) Structure and Performance of Cements, Taylor \& Francis, London, 2002.

[19] H.M. Jennings, Refinements to colloid model of C-S-H in cement: CM-II, Cement and Concrete Research, 38 (2008) 275-289.

[20] B. FelekoĞlu, K. Tosun, B. Baradan, A. Altun, Relationship between clinker porosity and interstitial phase morphology, Advances in Cement Research, 20 (2008) 109-119.

[21] H.S. Wong, N.R. Buenfeld, Euclidean Distance Mapping for computing microstructural gradients at interfaces in composite materials, Cement and Concrete Research, 36 (2006) 1091-1097.

[22] B. Felekoğlu, Effects of PSD and surface morphology of micro-aggregates on admixture requirement and mechanical performance of micro-concrete, Cement and Concrete Composites, 29 (2007) 481-489.

[23] Y. Gao, G. De Schutter, G. Ye, Micro- and meso-scale pore structure in mortar in relation to aggregate content, Cement and Concrete Research, 52 (2013) 149-160.

[24] M. Luković, B. Šavija, H. Dong, E. Schlangen, G. Ye, Micromechanical study of the interface properties in concrete repair systems, Journal of Advanced Concrete Technology, 12 (2014) 320-339.

[25] A. Attari, C. McNally, M.G. Richardson, A combined SEM-Calorimetric approach for assessing hydration and porosity development in GGBS concrete, Cement and Concrete Composites, 68 (2016) 46-56.

[26] H.S. Wong, K. Matter, N.R. Buenfeld, Estimating the original cement content and water-cement ratio of Portland cement concrete and mortar using backscattered electron microscopy, Magazine of Concrete Research, 65 (2013) 693 706.

[27] H.S. Wong, R.W. Zimmerman, N.R. Buenfeld, Estimating the permeability of cement pastes and mortars using image analysis and effective medium theory, Cement and Concrete Research, 42 (2012) 476-483.

[28] M.A.B. Promentilla, S.M. Cortez, R.A.D. Papel, B.M. Tablada, T. Sugiyama, Evaluation of Microstructure and Transport Properties of Deteriorated Cementitious Materials from Their X-ray Computed Tomography (CT) Images, Materials, 9 (2016) 388.

[29] D.W. Hadley, W.L. Dolch, S. Diamond, On the occurrence of hollow-shell hydration grains in hydrated cement paste, Cement and Concrete Research, 30 (2000) 1-6.

[30] M.K. Head, H.S. Wong, N.R. Buenfeld, Characterisation of 'Hadley' grains by confocal microscopy, Cement and Concrete Research, 36 (2006) 1483-1489.

[31] E. Gallucci, P. Mathur, K. Scrivener, Microstructural development of early age hydration shells around cement grains, Cement and Concrete Research, 40 (2010) 4-13.

[32] R.C. Smith, J.D. Carey, R.D. Forrest, S.R.P. Silva, Effect of aspect ratio and anode location on the field emission properties of a single tip based emitter, Journal of Vacuum Science \& Technology B, 23 (2005) 632-635. 


\section{APPENDIX}

This section presents the calculations of the probe diameters for the four different emitters used in the study (tungsten thermionic $(\mathrm{T}-\mathrm{W})$, lanthanum hexaboride thermionic $\left(\mathrm{T}-\mathrm{LaB}_{6}\right)$, zirconium oxide/tungsten Schottky field emitter (SFE) and tungsten cold field (CFE) emitters). The calculations are based on the method of [15] which introduces the concept of practical brightness $\left(\mathrm{B}_{\text {prac }}\right)$.

For thermionic and Schottky emitters:

$$
B_{\text {prac }}=1.44 \frac{e J}{\pi k T}
$$

and for cold field emitters:

$$
B_{\text {prac }}=1.44 \frac{e J}{\pi d}
$$

where $e$ is the elementary electric charge $\left(=1.60 \times 10^{-19} \mathrm{C}\right), j\left(\mathrm{~A} / \mathrm{m}^{2}\right)$ is the emission current density, $k$ is the Boltzmann constant $\left(=1.38 \times 10^{-23} \mathrm{~J} / \mathrm{K}\right), T(\mathrm{~K})$ is the emission temperature and $d(\mathrm{~J})$ is the mean tangential energy. The emission temperatures for T-W, T-LaB 6 and SFE are taken as 2700, 1700 and $1800 \mathrm{~K}$ respectively whereas the emission current density $j$ can be calculated using the following equations:

For thermionic emitters:

$$
J=-\left(\frac{4 \pi e m}{h^{3}}\right) k T^{2} \exp \left(\frac{-e \phi}{k T}\right)
$$

for Schottky field emitters:

$$
J=\frac{e m}{2 \pi^{2} \hbar^{3}}(k T)^{2} \exp \left(-\frac{\phi-\sqrt{\frac{e^{3} F}{4 \pi \varepsilon_{0}}}}{k T}\right) \frac{\pi q}{\sin \pi q}, \text { with } q=\frac{\hbar}{\pi k T \sqrt{m}}\left(4 \pi \varepsilon_{0} e F^{3}\right)^{1 / 4}
$$

And for cold field emitters:

$$
J=\frac{q^{2}}{8 \pi h \phi}(\beta F)^{2} \exp \left(-\frac{\frac{4}{3} \sqrt{\frac{8 \pi^{2} m}{h^{2}} \frac{(q \phi)^{3 / 2}}{q}}}{\beta F}\right)
$$

Where $m$ is the mass of electron $\left(=9.11 \times 10^{-31} \mathrm{~kg}\right), h$ is the Planck's constant $\left(=6.63 \times 10^{-31} \mathrm{Js}\right), \phi$ is the work function $\left(=4.5 \mathrm{eV}\right.$ for $\mathrm{W}, 2.4 \mathrm{eV}$ for $\mathrm{LaB}_{6}$ and $2.9 \mathrm{eV}$ for $\left.\mathrm{ZrO} / \mathrm{W}\right), \hbar$ is the reduced Planck's constant $\left(=1.05 \times 10^{-31} \mathrm{Js}\right), F$ is the electric field (taken as $6.5 \times 10^{8} \mathrm{~V} / \mathrm{m}$ for SFE and $10 \times 10^{8} \mathrm{~V} / \mathrm{m}$ for CFE) and $\varepsilon_{0}$ is the permittivity of free space $(=8.85$ $\left.\times 10^{-12} \mathrm{~F} / \mathrm{m}\right) . \beta$ is the field enhancement factor for CFE and it can calculated based on the geometry of the emitter tip using the equation proposed by [32]:

$$
\beta=1+\sqrt{h / 2 r}
$$

Note that this equation is based on the assumption that the distance between the anode plane and the emitter tip is sufficiently large. Supposing that the height $(h)$ and radius $(r)$ of the emitter tip are 4000 and $100 \mathrm{~nm}$ respectively, the resulting $\beta$ is equal to 5.47. Following the work of Wong and Buenfeld [10], it is assumed that the microscope is set up to image an atomic number contrast level of $2.5 \%$ with a detector collection efficiency of 0.1 and scan time of $100 \mathrm{~s}$ for a $1024 \times 768$ image. Thus, a probe current $\left(I_{p}\right)$ of $0.5 \mathrm{nA}$ is employed. By knowing the practical brightness and the probe current, the full width median (FW50) source image size can be determined as follows:

$$
d_{I}=\sqrt{\frac{4 I_{p}}{\pi^{2} \alpha_{p}^{2} V_{p} B_{p r a c}}}
$$


Where $\alpha_{p}$ and $V_{p}$ are the beam half opening angle at the target $(=0.005 \mathrm{rad})$ and the accelerating voltage $(5$ to $30 \mathrm{keV})$ respectively. The contributions from other sources including diffraction and chromatic and spherical aberrations are calculated using Eq. (I.8-1.10) respectively:

$$
\begin{aligned}
& d_{A}=\frac{0.66 \times 10^{-9}}{\sqrt{V_{p} \alpha_{p}}} \\
& d_{C}=0.6 C_{C} \frac{\Delta E_{F W 50}}{V_{p}} \\
& d_{S}=0.18 C_{S} \alpha_{p}^{3}
\end{aligned}
$$

Where $C_{c}(\mathrm{~m}), C_{s}(\mathrm{~m})$ are the chromatic and spherical aberration coefficients respectively and $\Delta E_{F W 50}(\mathrm{eV})$ is the FW50 variation in beam voltage. The $C_{c}$ and $C_{s}$ values for the thermionic emitters, SFE and CFE are taken as $0.01 \& 0.02 \mathrm{~m}$, $0.0027 \& 0.0032 \mathrm{~m}$ and $0.0018 \& 0.0020 \mathrm{~m}$ respectively whereas the $\Delta E_{F W 50}$ for T-W, T-LaB 6 , SFE and CFE are taken as $2,1,0.6$ and $0.3 \mathrm{eV}$ respectively. The total probe diameter $\left(d_{p}\right)$ is then calculated by adding all the contributions together using the root-power-sum (RPS) based equation:

$$
d_{p}=\sqrt{\left[\left(d_{A}^{4}+d_{S}^{4}\right)^{1.3 / 4}+d_{I}^{1.3}\right]^{2 / 1.3}+d_{C}^{2}}
$$




\section{TABLES \& FIGURES}

Table 1: Calculated practical brightness $\left(B_{\text {prac }}\right)$, diameter of source image $\left(d_{I}\right)$, contributions from chromatic (dc) and spherical $\left(d_{s}\right)$ aberrations and diffraction $\left(d_{A}\right)$, and resulting total probe diameter $\left(d_{p}\right)$ for different emitters at increasing accelerating voltages $(\mathrm{E})$. W, $\mathrm{LaB}_{6}$ and $\mathrm{ZrO}$ represent tungsten, lanthanum hexaboride and zirconium oxide respectively.

\begin{tabular}{|c|c|c|c|c|c|c|c|}
\hline & E (keV) & $B_{\text {pract }}\left(\mathbf{A} / \mathbf{m}^{2} \mathbf{s r}\right)$ & $d_{I}(n m)$ & $\mathbf{d}_{C}(\mathbf{n m})$ & ds (nm) & $d_{A}(n m)$ & $d_{p}(n m)$ \\
\hline \multirow{6}{*}{ 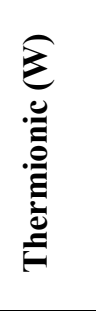 } & 5 & $3.44 \mathrm{E}+08$ & 153.54 & 6.80 & 0.45 & 1.87 & 154.07 \\
\hline & 10 & $6.88 \mathrm{E}+08$ & 108.57 & 3.40 & 0.45 & 1.32 & 108.89 \\
\hline & 15 & $1.03 \mathrm{E}+09$ & 88.65 & 2.27 & 0.45 & 1.08 & 88.90 \\
\hline & 20 & $1.38 \mathrm{E}+09$ & 76.77 & 1.70 & 0.45 & 0.93 & 76.98 \\
\hline & 25 & $1.72 \mathrm{E}+09$ & 68.66 & 1.36 & 0.45 & 0.83 & 68.85 \\
\hline & 30 & $2.06 \mathrm{E}+09$ & 62.68 & 1.13 & 0.45 & 0.76 & 62.85 \\
\hline \multirow{6}{*}{ 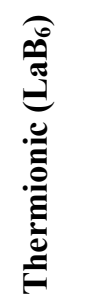 } & 5 & $4.17 \mathrm{E}+09$ & 44.09 & 3.40 & 0.45 & 1.87 & 44.78 \\
\hline & 10 & $8.34 \mathrm{E}+09$ & 31.18 & 1.70 & 0.45 & 1.32 & 31.62 \\
\hline & 15 & $1.25 \mathrm{E}+10$ & 25.46 & 1.13 & 0.45 & 1.08 & 25.80 \\
\hline & 20 & $1.67 \mathrm{E}+10$ & 22.05 & 0.85 & 0.45 & 0.93 & 22.34 \\
\hline & 25 & $2.08 \mathrm{E}+10$ & 19.72 & 0.68 & 0.45 & 0.83 & 19.98 \\
\hline & 30 & $2.50 \mathrm{E}+10$ & 18.00 & 0.57 & 0.45 & 0.76 & 18.24 \\
\hline \multirow{6}{*}{ 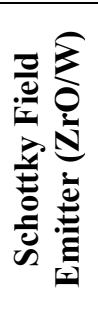 } & 5 & $2.35 \mathrm{E}+11$ & 5.88 & 0.55 & 0.07 & 1.87 & 6.89 \\
\hline & 10 & $4.69 \mathrm{E}+11$ & 4.16 & 0.28 & 0.07 & 1.32 & 4.87 \\
\hline & 15 & $7.04 \mathrm{E}+11$ & 3.39 & 0.18 & 0.07 & 1.08 & 3.97 \\
\hline & 20 & $9.38 \mathrm{E}+11$ & 2.94 & 0.14 & 0.07 & 0.93 & 3.44 \\
\hline & 25 & $1.17 \mathrm{E}+12$ & 2.63 & 0.11 & 0.07 & 0.83 & 3.08 \\
\hline & 30 & $1.41 \mathrm{E}+12$ & 2.40 & 0.09 & 0.07 & 0.76 & 2.81 \\
\hline \multirow{6}{*}{ 离 } & 5 & $3.41 \mathrm{E}+12$ & 1.54 & 0.18 & 0.05 & 1.87 & 2.91 \\
\hline & 10 & $6.83 \mathrm{E}+12$ & 1.09 & 0.09 & 0.05 & 1.32 & 2.06 \\
\hline & 15 & $1.02 \mathrm{E}+13$ & 0.89 & 0.06 & 0.05 & 1.08 & 1.68 \\
\hline & 20 & $1.37 \mathrm{E}+13$ & 0.77 & 0.05 & 0.05 & 0.93 & 1.45 \\
\hline & 25 & $1.71 \mathrm{E}+13$ & 0.69 & 0.04 & 0.05 & 0.83 & 1.30 \\
\hline & 30 & $2.05 \mathrm{E}+13$ & 0.63 & 0.03 & 0.05 & 0.76 & 1.19 \\
\hline
\end{tabular}




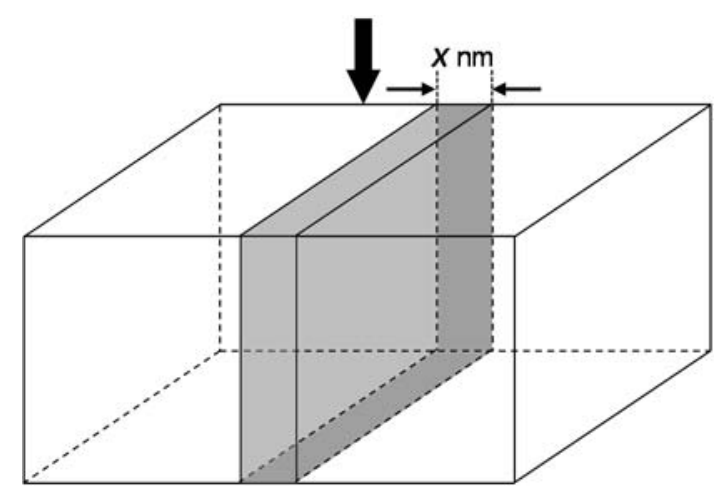

(Model A)

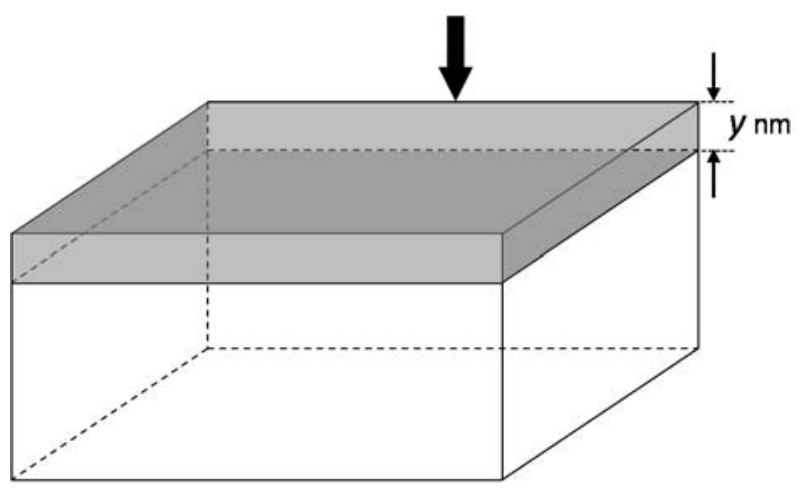

(Model B)

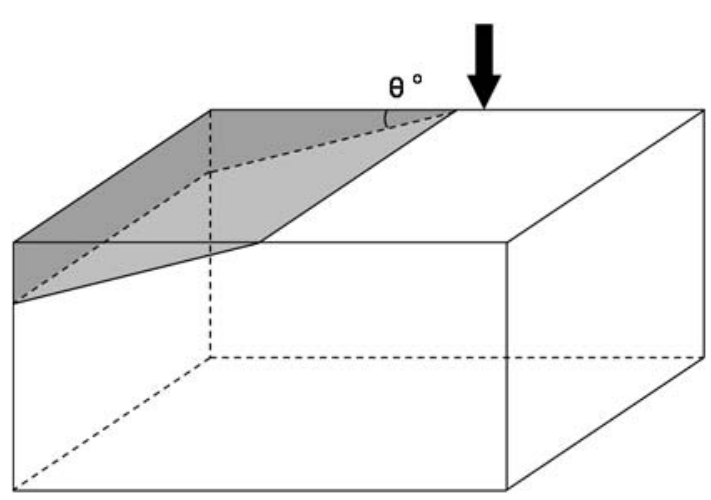

(Model C)

Figure 1. Schematics (not to scale) of the generated 3D pore-solid models for studying the effect of (A) pore size, (B) sampling subsurface materials and $(C)$ inclination angle of the pore-solid (C-S-H) boundary on BSE signal. Note that the shaded region represents epoxy-filled pore whereas the unshaded region represents $\mathrm{C}-\mathrm{S}-\mathrm{H}$. The direction of the electron beam is indicated by the black arrow. 


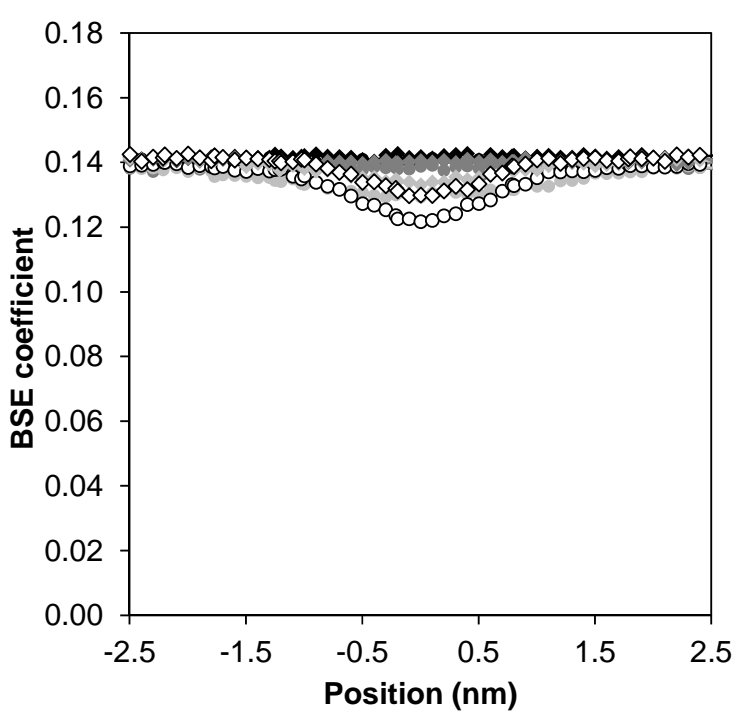

(a) $1 \mathrm{~nm}$ pore

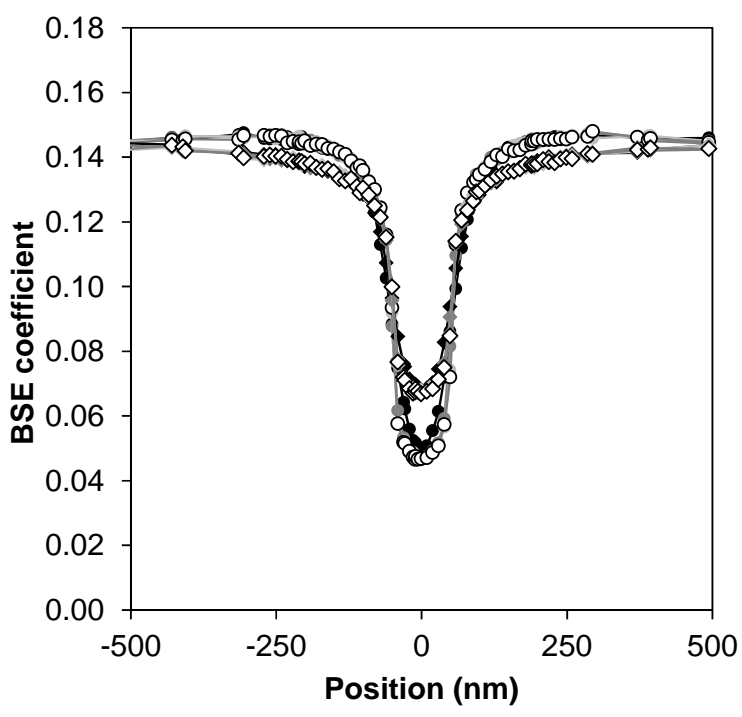

(c) $100 \mathrm{~nm}$ pore

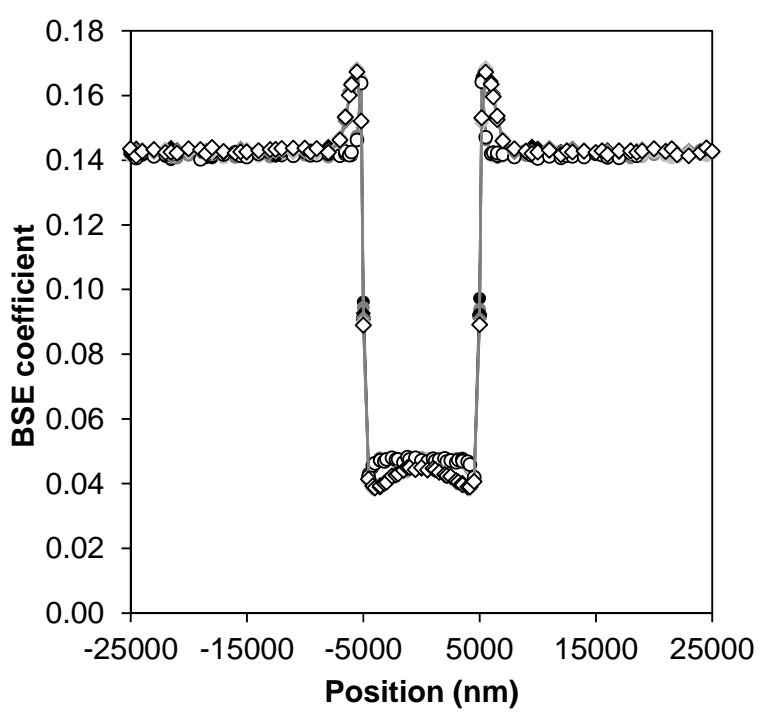

(e) $10 \mu \mathrm{m}$ pore

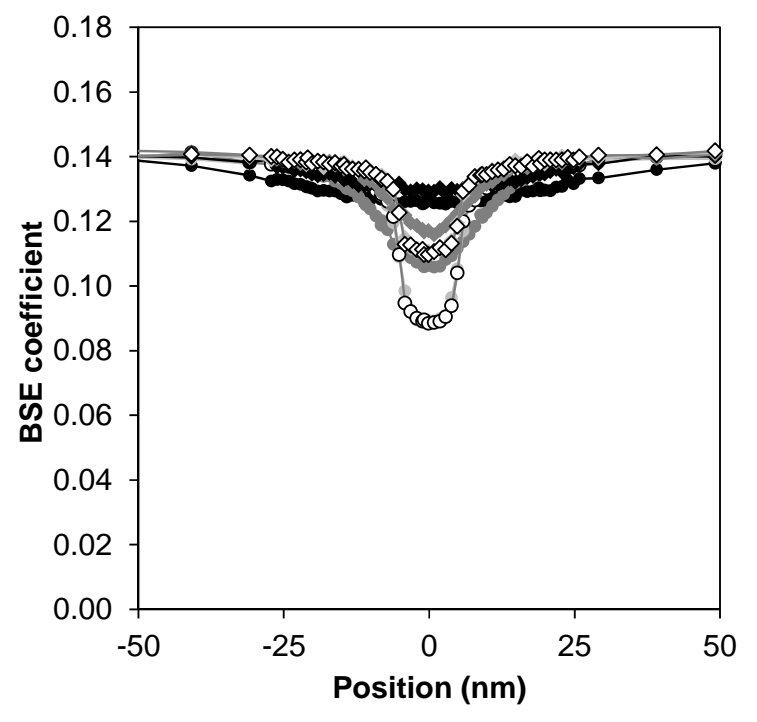

(b) $10 \mathrm{~nm}$ pore

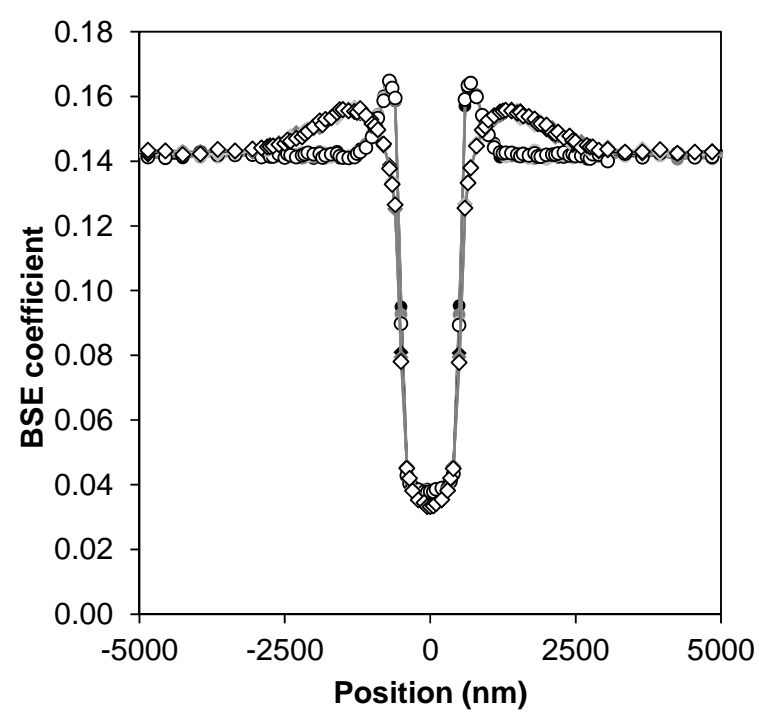

(d) $1 \mu \mathrm{m}$ pore

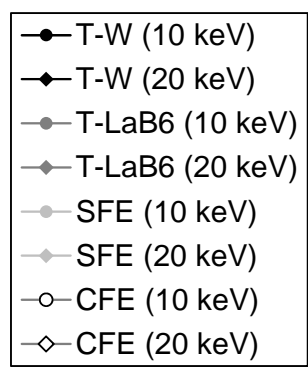

Figure 2. Variation in BSE coefficient across pore sizes of (a) $1 \mathrm{~nm},($ b) $10 \mathrm{~nm}$, (c) $100 \mathrm{~nm}$, (d) $1 \mu \mathrm{m}$ and (e) $10 \mu \mathrm{m}$ at 10 and $20 \mathrm{keV}$ for different emitters (Model $\mathrm{A}$ ). The pixel spacing is one tenth of the pore size. 


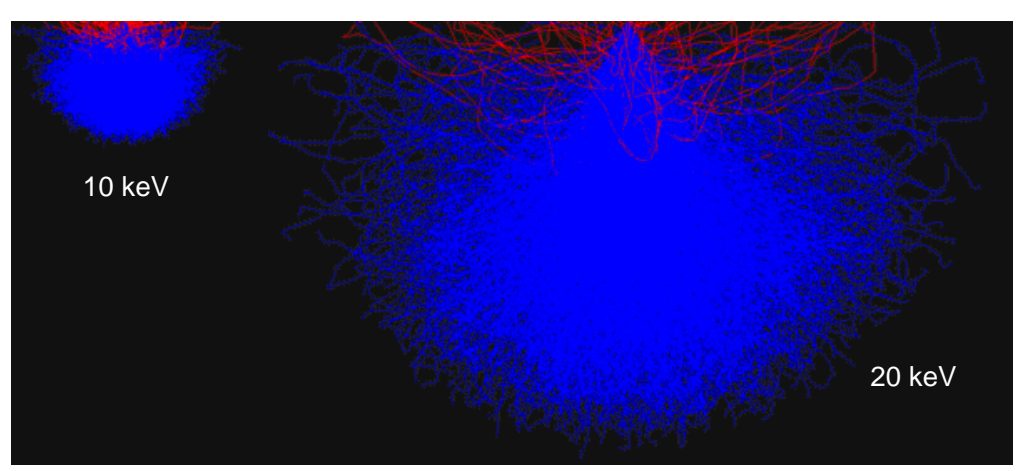

(a)

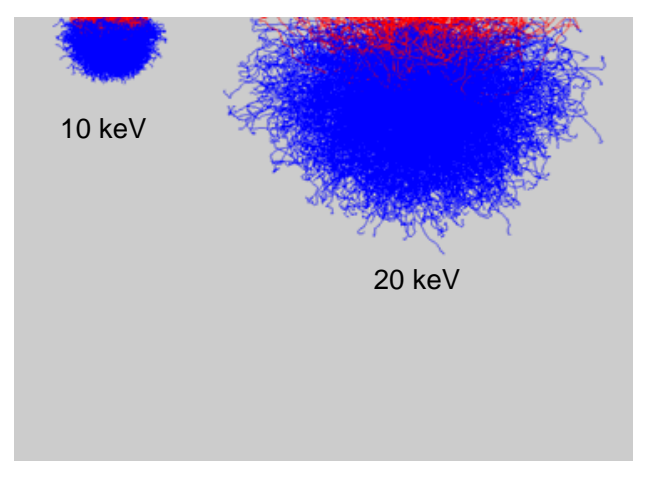

(b)

Figure 3. Monte Carlo simulations of electron-solid interactions in (a) pure epoxy and (b) pure C-S-H performed with a Schottky field emitter at 10 and $20 \mathrm{keV}$. Blue lines represent absorbed electron trajectories whereas red lines represent backscattered electron trajectories. Scale bar is $3 \mu \mathrm{m}$.

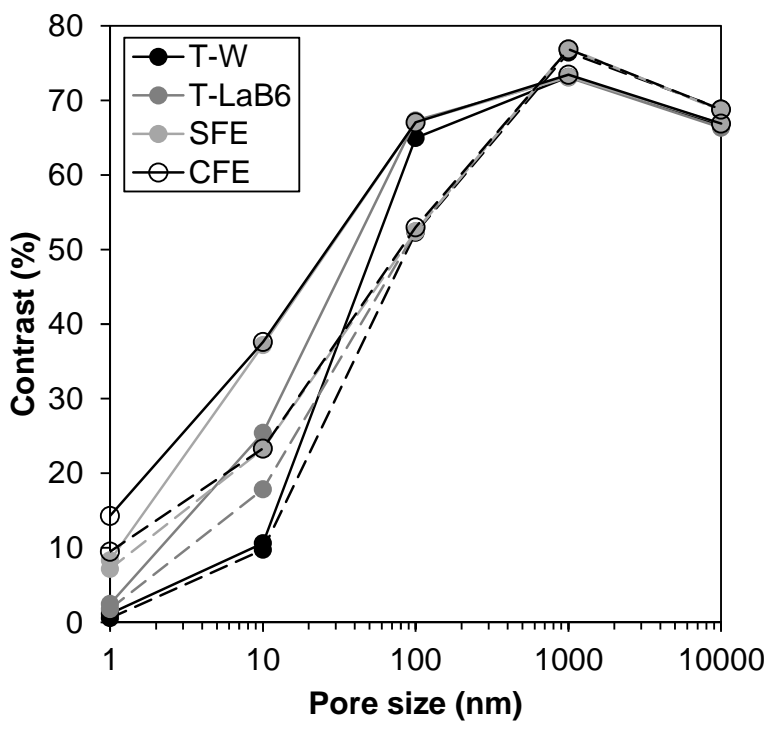

(a)

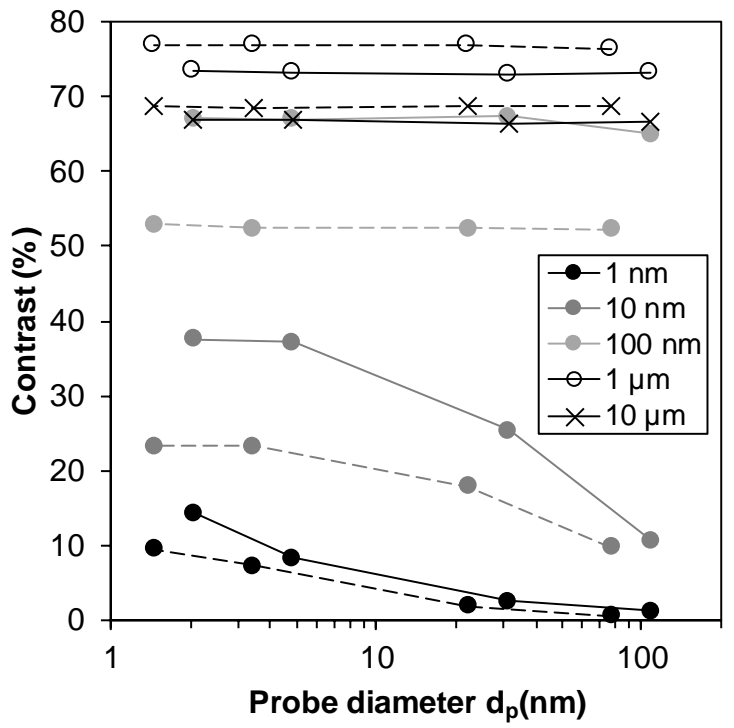

(b)

Figure 4. Calculated BSE contrast from Model A between the pore and C-S-H as a function of (a) pore size and (b) probe diameter at $10 \mathrm{keV}$ (solid line) and $20 \mathrm{keV}$ (dashed line). Values in the legend to (b) are pore size. 
T-W

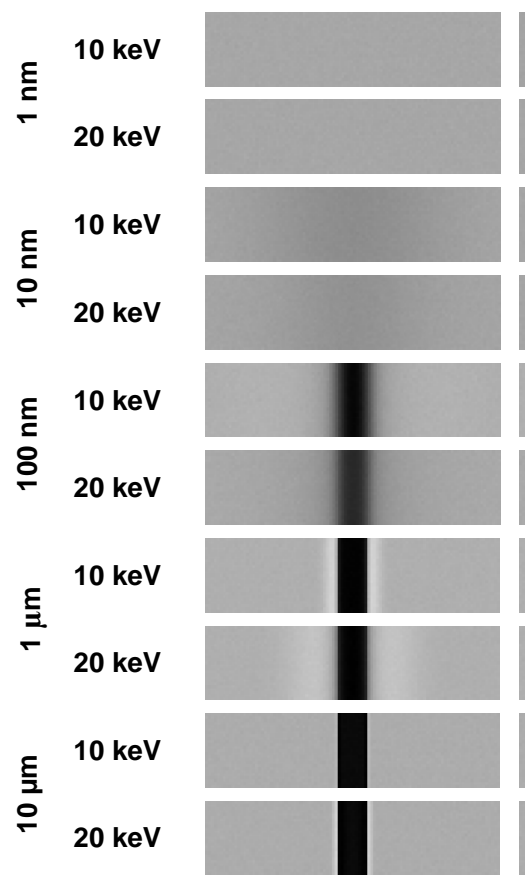

T-LaB 6
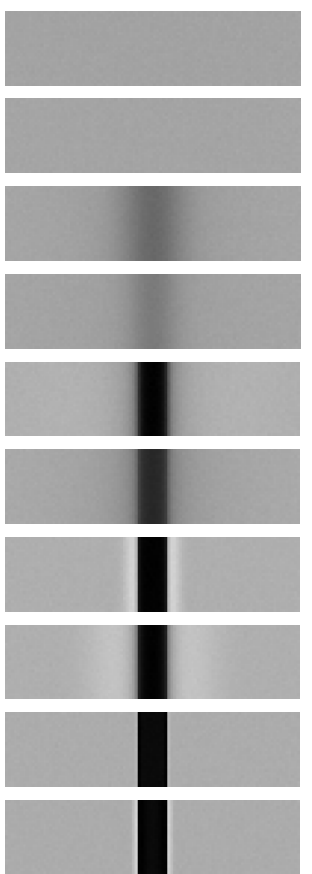

SFE
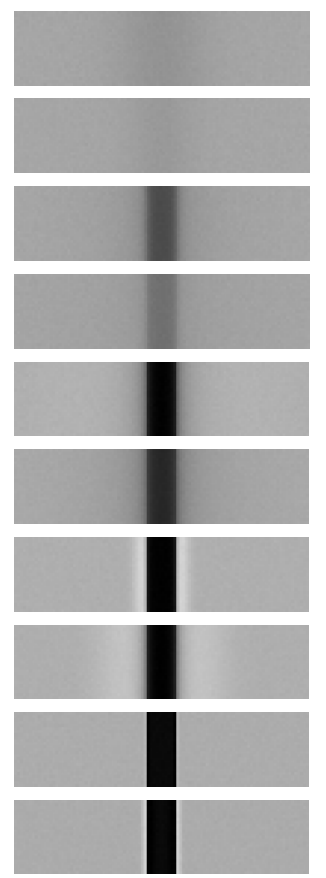

CFE

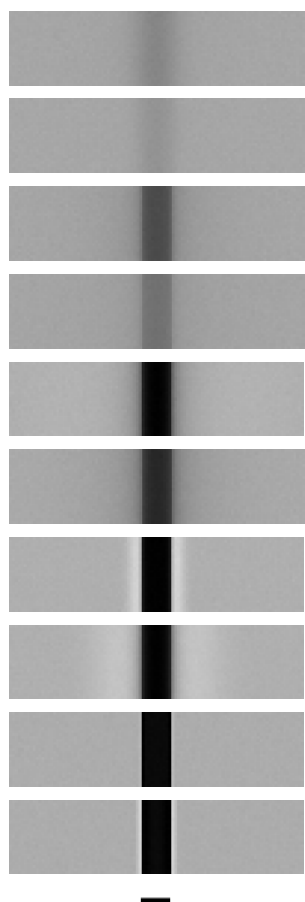

Figure 5. Simulated BSE images of pores (Model A) showing the effect of pore size, beam energy and emitter type on visibility of the pore. Pixel size is one-tenth of the pore size and image size is $100 \times 25$ pixels. Scale bar represents the pore size.

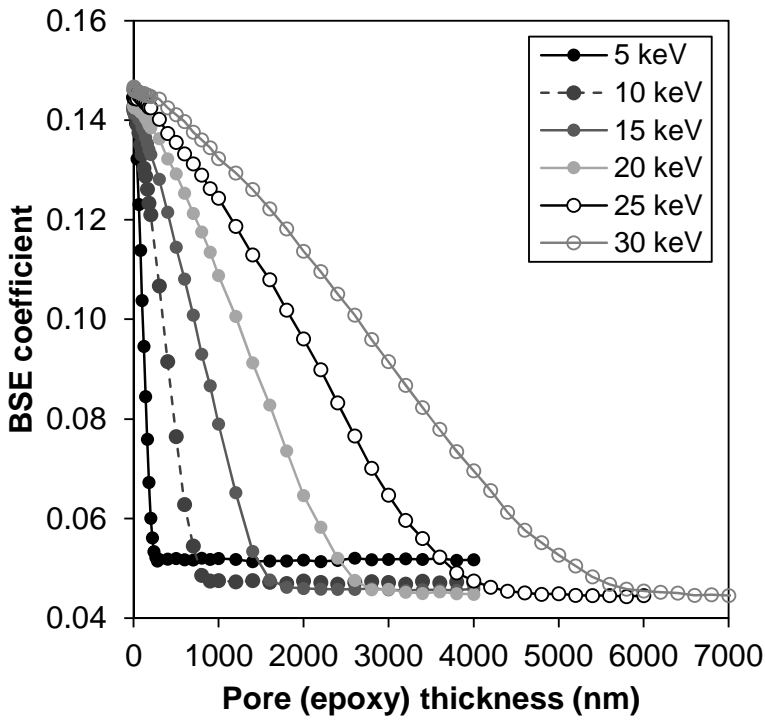

(a)

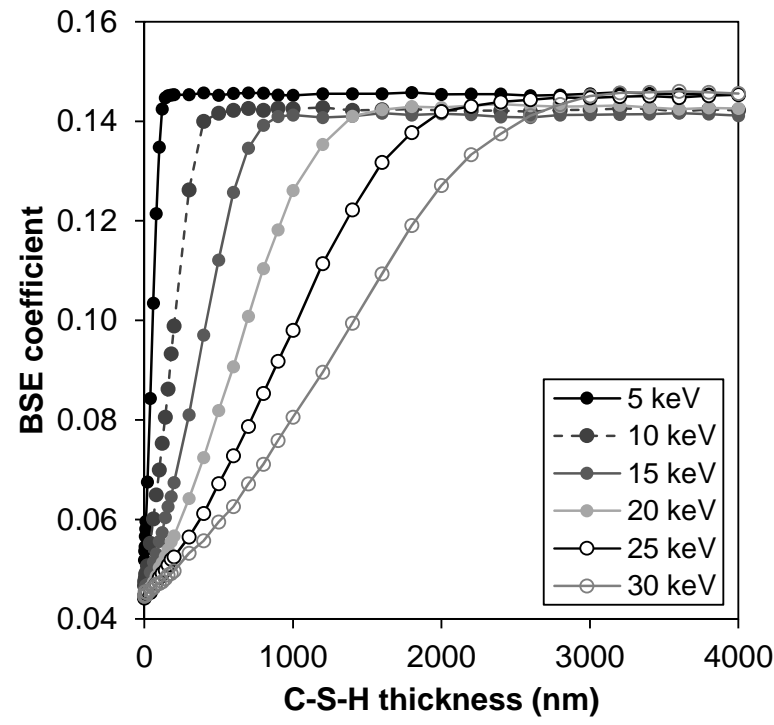

(b)

Figure 6. BSE coefficient changes as a function of the thickness of the top layer due to sampling of the bottom layer. Simulations were carried out on Model B for the case of (a) epoxy-filled pore layer overlying C-S-H layer and (b) C-S-H layer overlying epoxy-filled pore layer. 


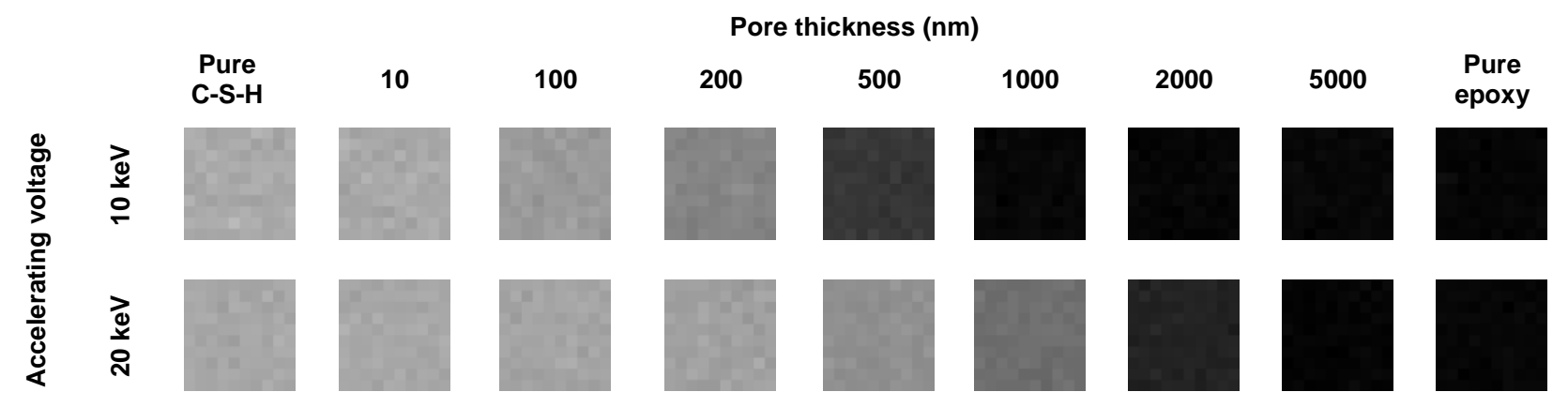

Figure 7. Simulated BSE images of an epoxy-filled pore that is overlying C-S-H. The resultant grey value of the pore varies depending on its thickness (i.e. depth). Grey values of pure epoxy and C-S-H are shown for comparison. Image size is $10 \times 10$ pixels at $0.1 \mu \mathrm{m}$ spacing.

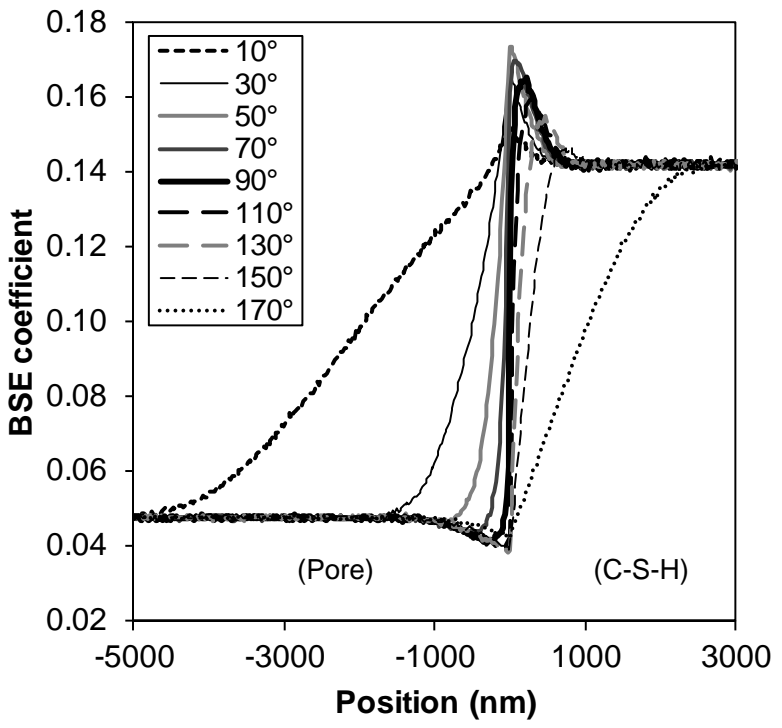

(a)

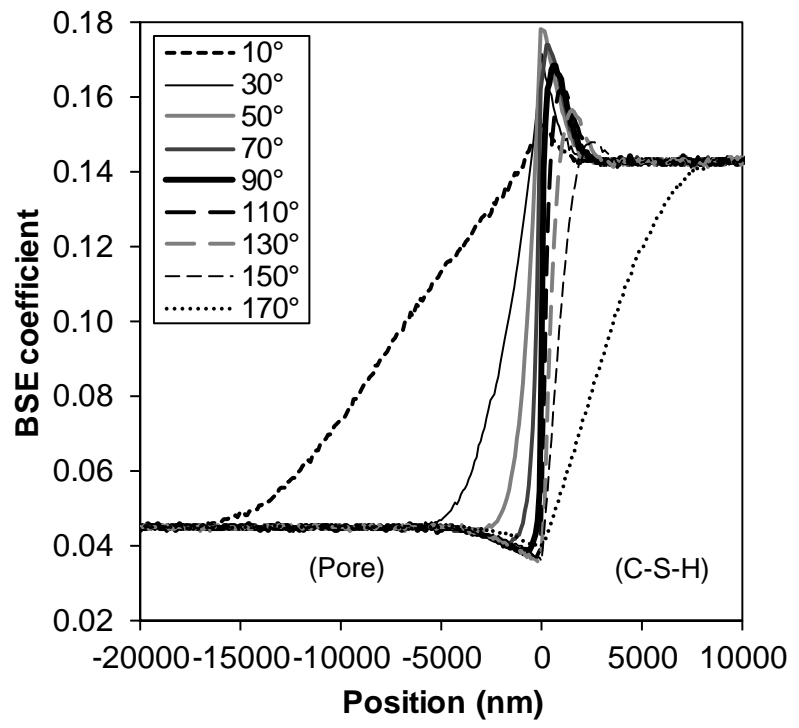

(b)

Figure 8. Change in BSE coefficient across pore-C-S-H boundaries set at various inclination angles (Model C) at (a) 10 $\mathrm{keV}$ and (b) $20 \mathrm{keV}$. 


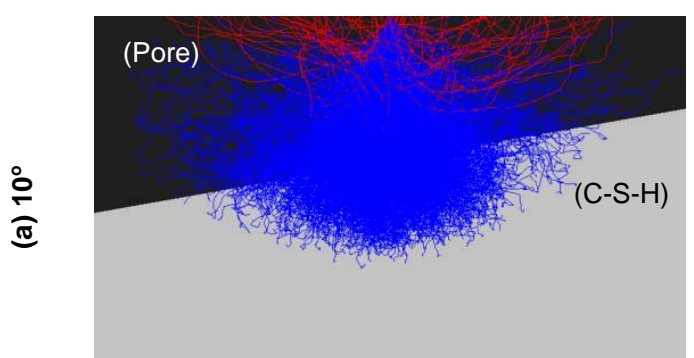

$-5000 \mathrm{~nm}$

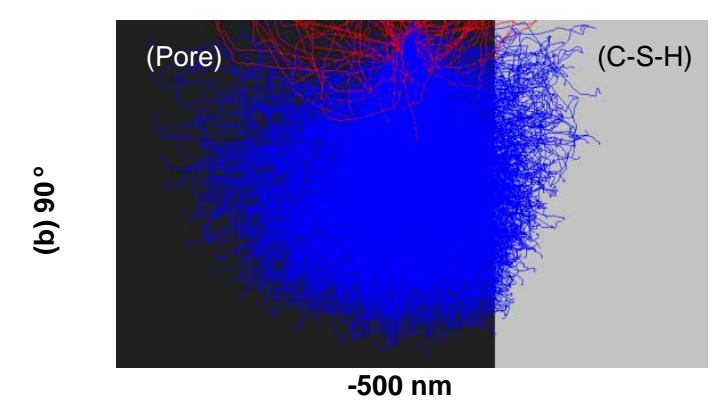

$-500 \mathrm{~nm}$

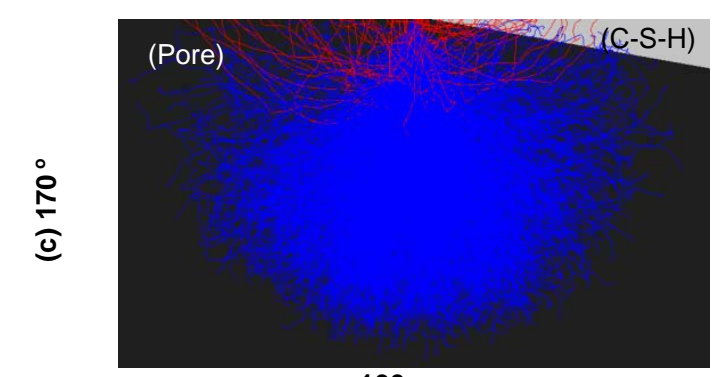

$-100 \mathrm{~nm}$

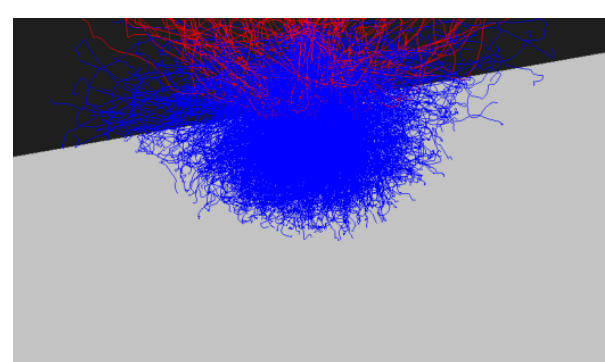

$-3000 \mathrm{~nm}$
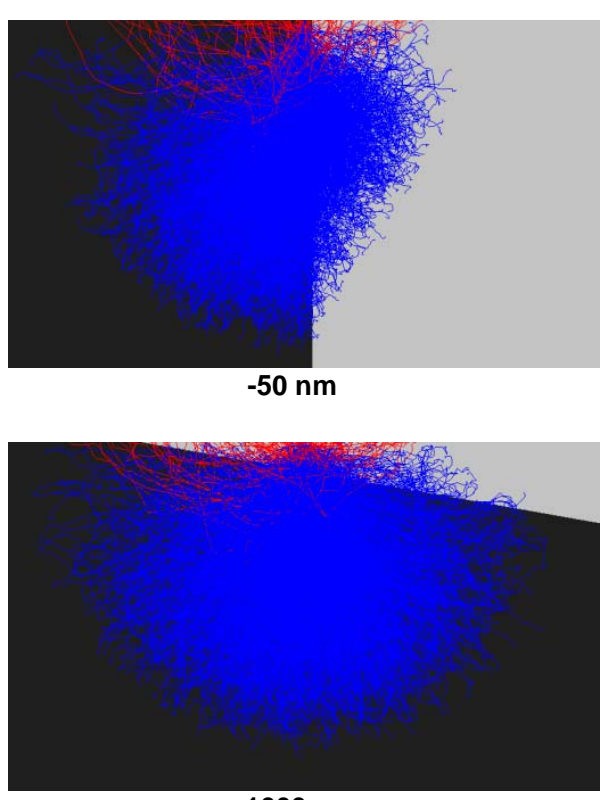

+1000 nm

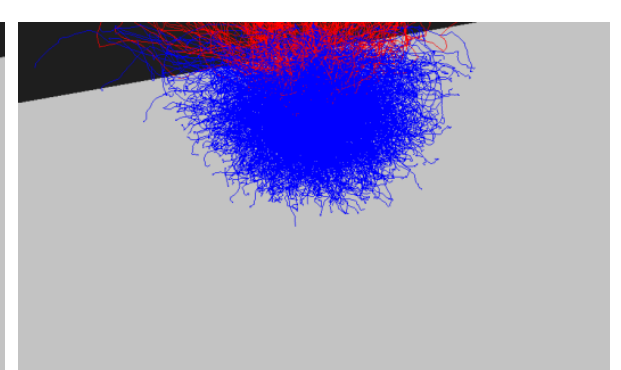

$-1000 \mathrm{~nm}$
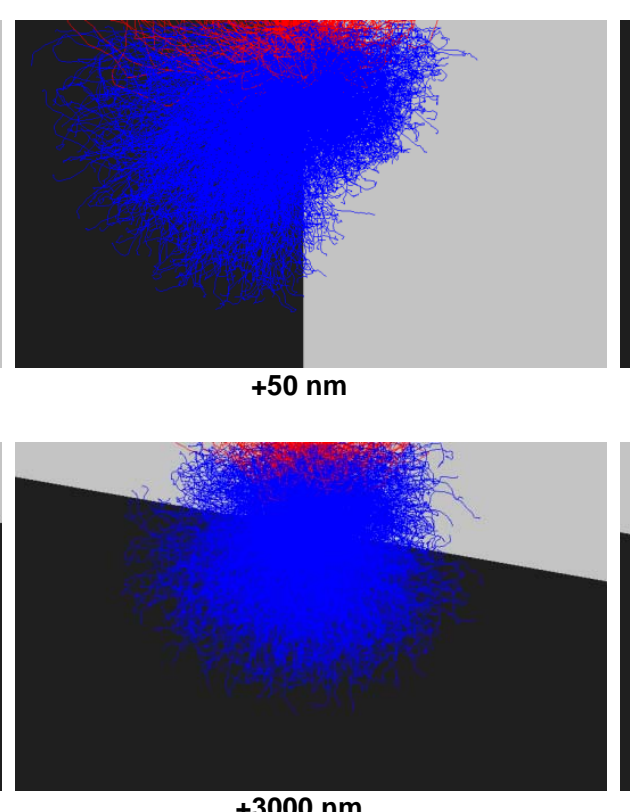

$+3000 \mathrm{~nm}$

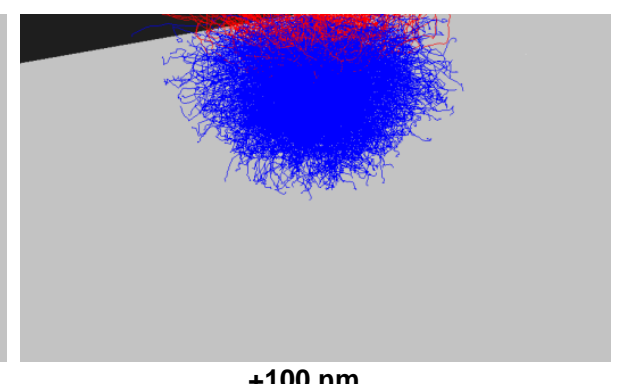

$+100 \mathrm{~nm}$

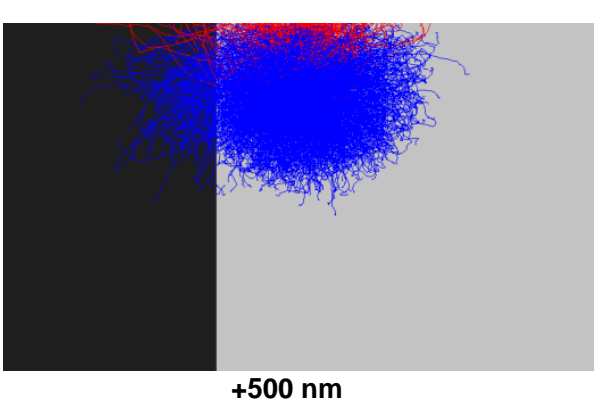

$+500 \mathrm{~nm}$

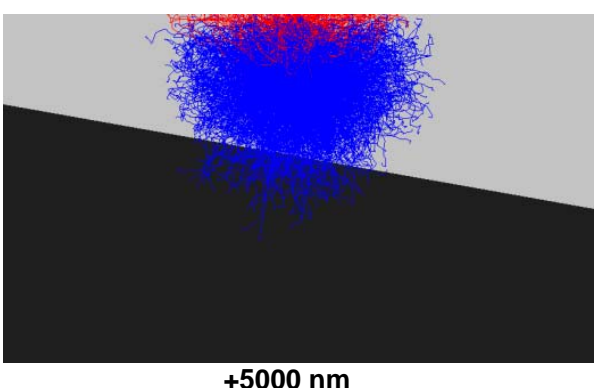

$+5000 \mathrm{~nm}$

Figure 9. Change in shape and size of the interaction volume as the beam is scanned across pore-C-S-H boundary set at inclination angles of (a) $10^{\circ}$, (b) $90^{\circ}$ and (c) $170^{\circ}$ at 10 keV. Note that the dark region represents epoxy-filled pore whereas the bright region represents $\mathrm{C}-\mathrm{S}-\mathrm{H}$. Scale bar is $1 \mu \mathrm{m}$. 


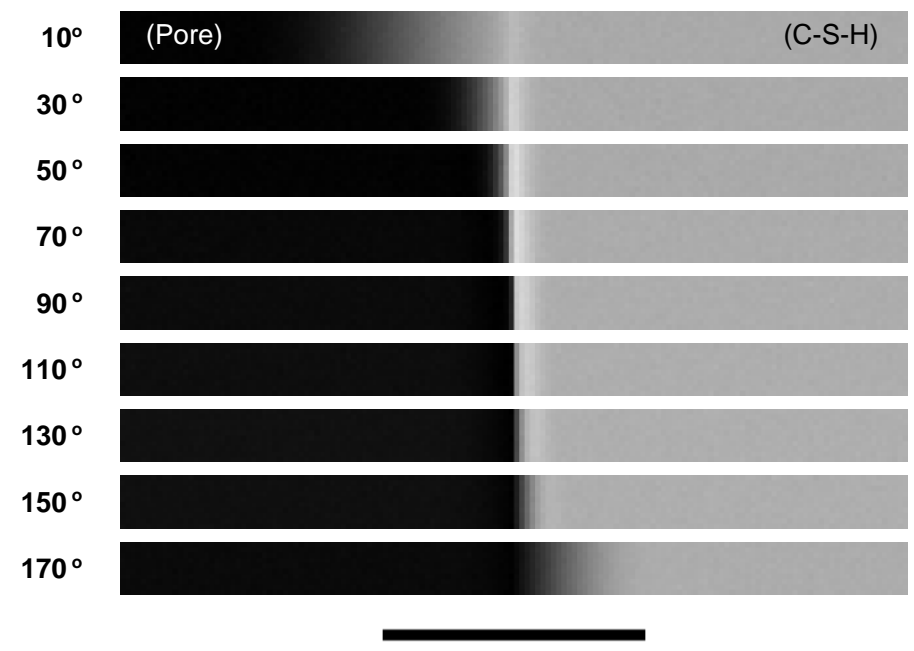

Figure 10. Simulated BSE images $(10 \mathrm{keV})$ across pore-C-S-H boundaries at various inclination angles showing grey value transition near boundary. Image size is $1800 \times 120$ pixels at $0.1 \mu \mathrm{m}$ spacing. Scale bar is $6 \mu \mathrm{m}$. 


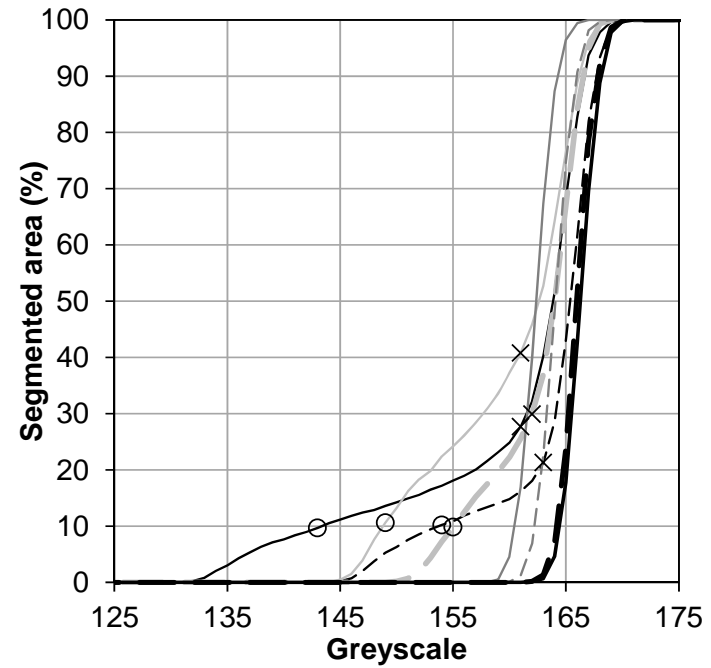

(a) $1 \mathrm{~nm}$ pore

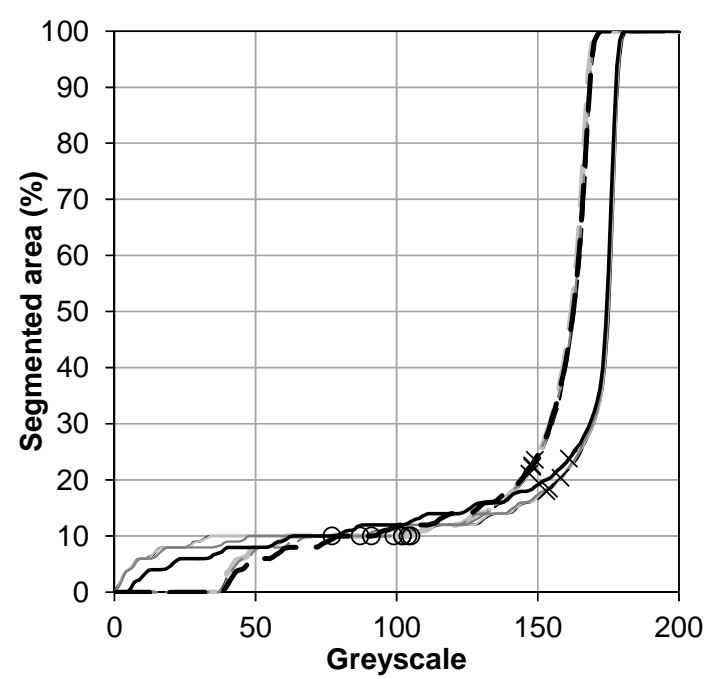

(c) $100 \mathrm{~nm}$ pore

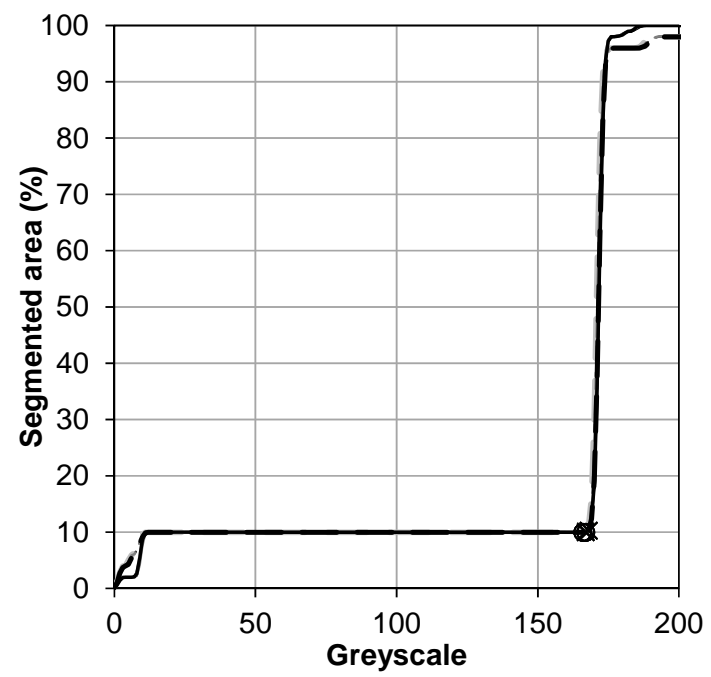

(e) $10 \mu \mathrm{m}$ pore

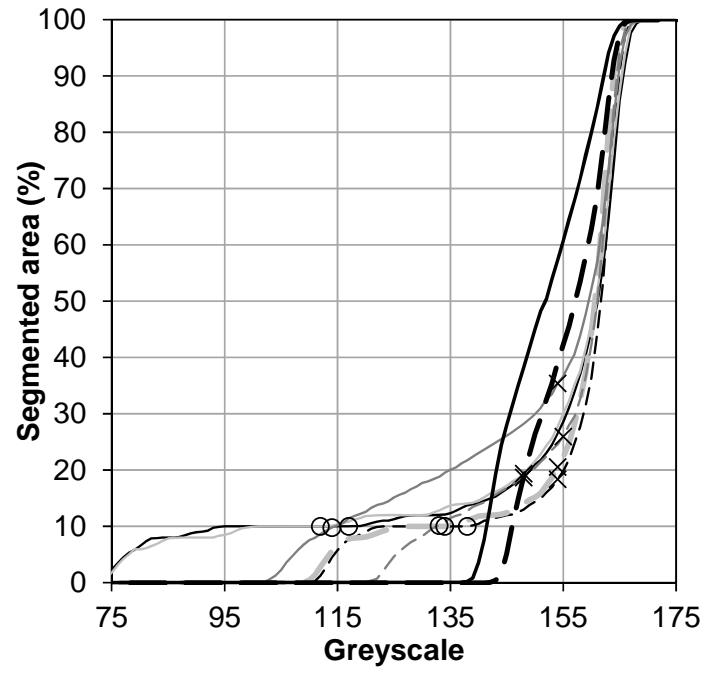

(b) $10 \mathrm{~nm}$ pore

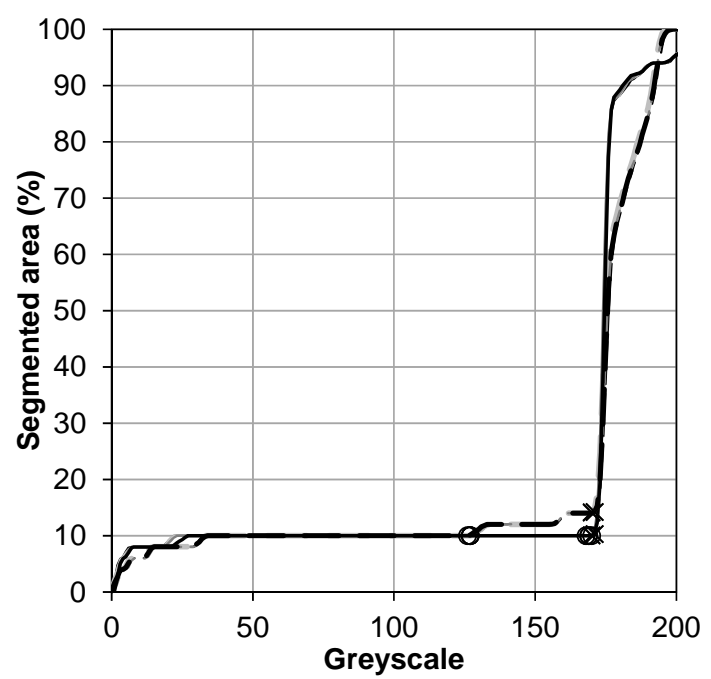

(d) $1 \mu \mathrm{m}$ pore

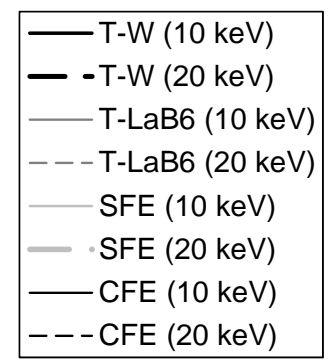

Figure 11. Cumulative brightness histogram for the simulated BSE images in Sections $3.1 \& 3.2$ (from Figure 5) at increasing pore size. Crosses mark the inflection point determined by the Overflow segmentation method whereas circles mark the correct grey values that give the actual pore fraction. Note that the scale of the $\mathrm{X}$-axis has been expanded for clarity. 


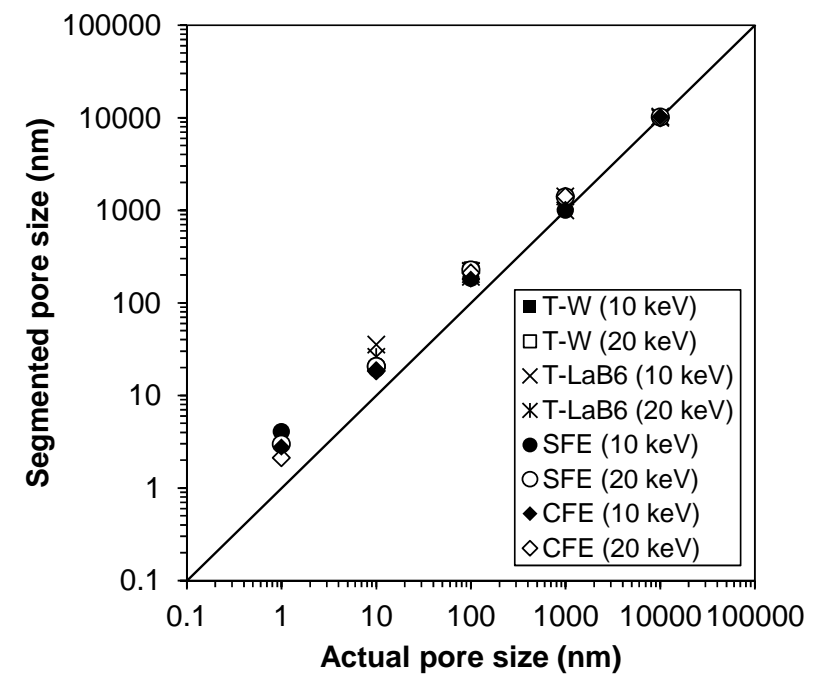

(a)

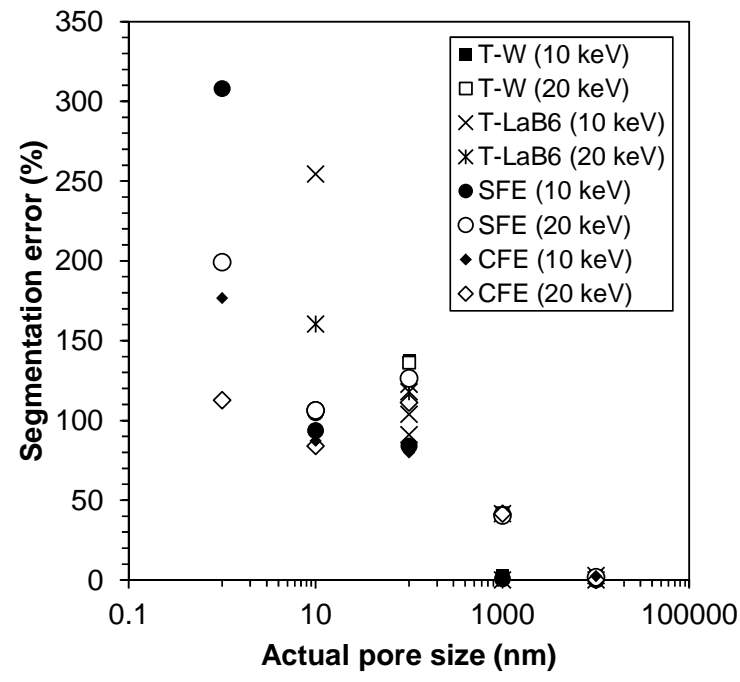

(b)

Figure 12. (a) Comparison between the Overflow segmented pore size and the actual pore size; (b) segmentation error vs. pore size.

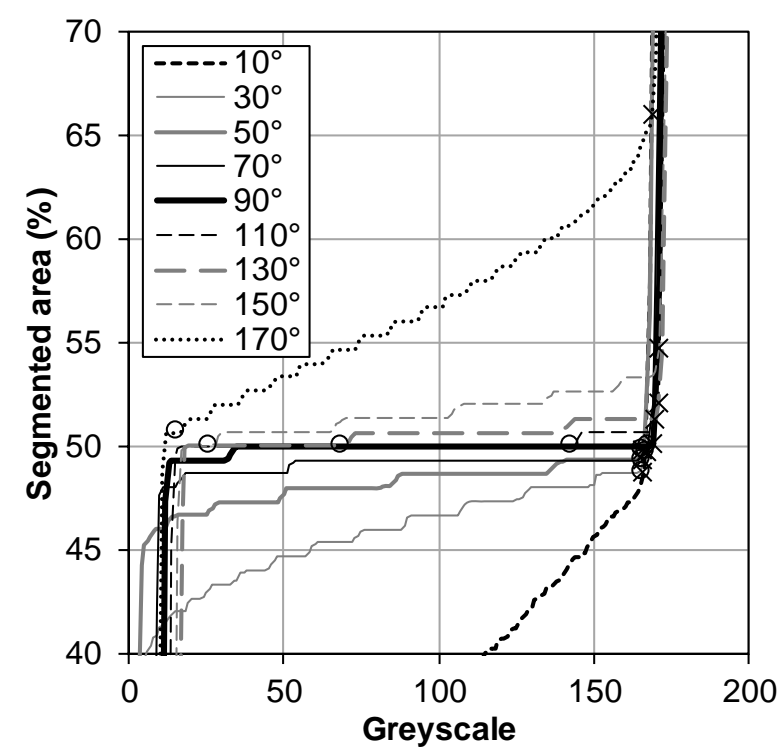

(a)

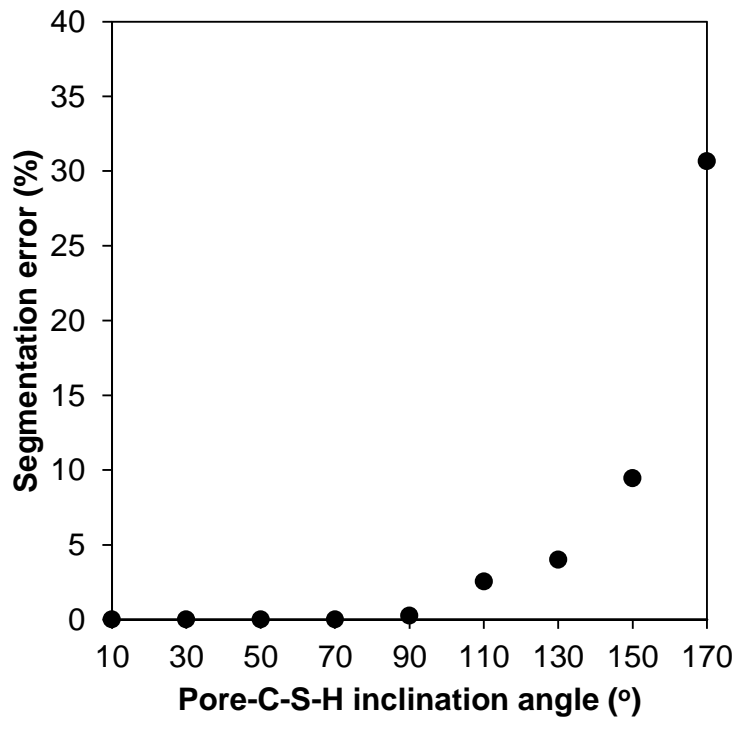

(b)

Figure 13. (a) Cumulative brightness histogram for the simulated BSE images in Section 3.4 (from Figure 10) at increasing pore boundary inclination angle. Crosses mark the inflection point determined by the Overflow segmentation method whereas circles mark the correct grey values that give the actual pore fraction. Note that the scales of the $\mathrm{Y}$ and $\mathrm{X}$-axes have been expanded for clarity; (b) segmentation error vs. pore-C-S-H boundary inclination angle. 